\title{
Generalized internal multiple imaging
}

\author{
M. A. H. Zuberi ${ }^{1}$ and Tariq Alkhalifah ${ }^{1}$
}

\begin{abstract}
Internal multiples deteriorate the image when the imaging procedure assumes only single scattering, especially if the velocity model does not have sharp contrasts to reproduce such scattering in the Green's function through forward modeling. If properly imaged, internal multiples (internally scattered energy) can enhance the seismic image. Conventionally, to image internal multiples, accurate, sharp contrasts in the velocity model are required to construct a Green's function with all the scattered energy. As an alternative, we have developed a generalized internal multiple imaging procedure that images any order internal scattering using the background Green's function (from the surface to each image point), constructed from a smooth velocity model, usually used for conventional imaging. For the first-order internal
\end{abstract}

multiples, the approach consisted of three steps, in which we first back propagated the recorded surface seismic data using the background Green's function, then crosscorrelated the back-propagated data with the recorded data, and finally crosscorrelated the result with the original background Green's function. This procedure images the contribution of the recorded first-order internal multiples, and it is almost free of the single-scattering recorded energy. The cost includes one additional crosscorrelation over the conventional single-scattering imaging application. We generalized this method to image internal multiples of any order separately. The resulting images can be added to the conventional single-scattering image, obtained, e.g., from Kirchhoff or reversetime migration, to enhance the image. Application to synthetic data with reflectors illuminated by multiple scattering (double scattering) demonstrated the effectiveness of the approach.

\section{INTRODUCTION}

Internal multiples recorded in the seismic data can be an invaluable source of information in cases in which the single-scattering approximation is limited to image certain reflectors. Moreover, the multiscattered data not only improve the illumination of the target reflectors but also enhance the amplitudes in the presence of strong scattering layers (like in Saudi Arabia, where strong scattering above target reflectors weakens their image). Conventionally, to image internal multiples, one would need sharp contrasts in the velocity model to build Green's functions that include the multiple scattering. However, often such sharp contrast information for the velocity model is not readily available.

Recently, an iterative imaging procedure has been suggested by Behura et al. (2012) (see also Behura et al., 2014; Slob et al., 2014) to image all the internal scatterings together with single scattering. This iterative process does not allow for separate imaging of different orders of multiples. It also requires muting of anticausal events after crosscorrelation, but this can be automated. Slob et al. (2014) take a different route, in that they decompose the focusing function into up- and downgoing parts. The Marchenko method of inverse scattering is considered applicable to acoustic fields; therefore, the Marchenko-type methods such as those mentioned above should give correct amplitudes. In this study, we do not rely on the Marchenko equation. Other developments in imaging multiple scattering are given by Malcolm et al. (2011), who image prismatic waves and other higher order internal scatterings. In their work, the scatterers are separated into regions according to illumination decomposition. This decomposition splits each term of the Born scattering series into further terms, some of which are ignored. The ignored terms are the ones for which the wavefront sets of velocity perturbations have no points in common. In contrast, we propose to directly work with the Born series; i.e., we do not make any assumptions about the geometry of velocity perturbations. Moreover, our method differs from that described by Malcolm et al. (2009) in the implementation algorithm. Malcolm et al. (2009) project the full

Manuscript received by the Editor 29 July 2013; revised manuscript received 28 January 2014; published online 5 August 2014.

${ }^{1}$ King Abdullah University of Science and Technology, Thuwal, Saudi Arabia. E-mail: mohammad.zuberi@kaust.edu.sa; tariq.alkhalifah@kaust.edu.sa.

(C) 2014 Society of Exploration Geophysicists. All rights reserved. 
data into different orders of internal multiples by optimizing the $L^{2}$ norm of the difference between the full data and the desired order of internal multiples, whereas in our method we do not use any optimization.

In this study, we propose a generalized internal multiple imaging (GIMI) process, which would image any order of internal scattering, separately. We also prove that the conventional reverse-time migration (RTM) images first-order multiple scattering (the double bounce), but relative to the single scattering it tends to be weaker in amplitude. In this GIMI process, we rely solely on the background Green's function based on the conventional smooth velocity model. The additional computational cost is just a crosscorrelation of the surface seismic data with the back-propagated data. The number of crosscorrelations required is one less than the order of the term in the Born scattering series (or equal to the order of internal multiple) that we intend to image. Although the theoretical derivation given here for GIMI applies to terms of all orders of the Born scattering series, numerical examples of only the first two terms (single-scattering and first-order internal multiples) are shown in the examples section.

We also discuss the limitations of GIMI, namely, crosstalk and weak scatterers. The crosstalk is the result of the additional crosscorrelations with the surface data and, thus, can be mitigated by a simple procedure. A small velocity perturbation assumption is, however, necessary for the convergence of the Born scattering series. GIMI could be implemented either by the wavefield extrapolation method or more efficiently by the integral method, which involves only crosscorrelations and summations of the surface data (such as Kirchhoff migration). In this study, we use the integral method for imaging. When using the integral method, we do not have to worry about the scatterer strength (especially when we are ignoring the amplitudes) because all the scatterings are contained in the data. A nonconvergent Born series would mean that we would have internal multiples in the recorded data that are higher in amplitude than the primaries, but this is never the case. Even if internal multiples in the data have higher amplitudes than primaries, GIMI would image them, but the crosstalk noise will be very high and the simple procedure described in this study would not work. Therefore, as long as the data have internal multiples lower in amplitude than primary reflections, the crosstalk suppression procedure described in this study would work.

For the first-order internal multiple imaging (second-order term in the Born scattering series), GIMI can be described as a three-step procedure (Zuberi and Alkhalifah, 2014). The first step consists of the back propagation of the recorded surface data using the background Green's function. In the second step, we crosscorrelate the back-propagated data with the surface data. The final step consists of crosscorrelating the result of second step with the background Green's function from the surface to the image point. This resultant image contains enhanced first-order internal multiple contribution, whereas the single-scattering contribution has been suppressed. In other words, we have selected the first-order internal multiple for imaging. We can add this image to the result from conventional single-scattering imaging, e.g., Kirchhoff migration, to give the total image. The generalized method is obtained by repeating the second step of the three-stage procedure. In the next section, we will present the theoretical background of the proposed method.

\section{THEORY}

In the following derivation, we will focus on the phase aspect of imaging ignoring the amplitude. Throughout the derivation, the location of an image point in the subsurface is described by the Cartesian coordinate vector $\mathbf{X}$, whereas $\mathbf{x}$ with and without numerical subscripts, e.g., $\mathbf{x}_{0}$, are points on the surface (the recording surface). Vectors with primes as superscripts, e.g., $\mathbf{x}^{\prime}$, will be in the volume enclosed by the surface of integration. All the derivations are in the frequency domain including the resultant images represented by $\mathbf{I}$. Subscripts on $\mathbf{I}$ represent the order of the imaged term in the Born scattering series; e.g., $\mathbf{I}_{2}$ represents the image corresponding to second-order term in the Born series or first-order internal multiples. After imaging, a summation over the frequency is implied because we adhere to the zero-time imaging condition.

Although direct arrivals can be useful in imaging under certain circumstances (Zuberi and Alkhalifah, 2013), here, we shall ignore them. Surface multiples can also add to the quality of the image (double illumination discussed by Schuster et al., 2004), and they can also be used to fill in the missing data (Shan and Guitton, 2004). Nonetheless, surface multiples still cannot illuminate areas that are not illuminated by single scattering. This can be seen by considering a vertical reflector. A wave coming from the surface and incident on a vertical reflector at any angle cannot go back to the surface unless it bounces off another interface. Therefore, we shall assume that free-surface multiples have been eliminated from the data and ignore them in our study.

\section{Conventional imaging (with smooth velocity)}

The scattered field, based on the Lippmann-Schwinger equation, for an acoustic medium with constant density (which without loss of generality can be set to one) is given by (Ikelle and Amundsen, 2005)

$$
G_{s}\left(\mathbf{x}, \mathbf{x}_{0}\right)=\omega^{2} \int_{V} G_{0}\left(\mathbf{x}, \mathbf{x}^{\prime}\right) \delta v\left(\mathbf{x}^{\prime}\right) G\left(\mathbf{x}^{\prime}, \mathbf{x}_{0}\right) d^{3} \mathbf{x}^{\prime}
$$

where $G, G_{s}$, and $G_{0}$ are the total, scattered, and background Green's functions, respectively. Also, note that the LippmannSchwinger equation is implicit in $G$; i.e., $G=G_{0}+G_{S}$. The velocity perturbation is represented by $\delta v\left(\mathbf{x}^{\prime}\right)$. Equation 1 represents a sum of the scattered field from a source at $\mathbf{x}_{0}$, scattered from the points $\mathbf{x}^{\prime}$ and recorded at $\mathbf{x}$. The total field, based on the crosscorrelation interferometric theory, also satisfies the following relation (Wapenaar and Fokkema, 2006):

$$
G^{*}\left(\mathbf{x}, \mathbf{x}_{0}\right)+G\left(\mathbf{x}, \mathbf{x}_{0}\right) \approx \int_{\partial V} G^{*}\left(\mathbf{x}, \mathbf{x}^{\prime \prime}\right) G\left(\mathbf{x}^{\prime \prime}, \mathbf{x}_{0}\right) d^{2} \mathbf{x}^{\prime \prime}
$$

Equation 2 is equation 31 in Wapenaar and Fokkema (2006), in which the scaling factor involving surface velocity and density in that equation has been ignored. It can also be thought as being absorbed in the approximation in equation 2 because we do not care about absolute amplitudes in this study. Let $G_{\text {back }}$ be the wavefield obtained by back propagating the surface data. Using the background Green's function and equations 1 and 2, we extrapolate our recorded data to the potential image points in the subsurface, and we obtain $G_{\text {back }}$ as follows: 


$$
\begin{aligned}
\underbrace{\int_{\partial V} G_{s}\left(\mathbf{x}, \mathbf{x}_{0}\right) G_{0}^{*}(\mathbf{x}, \mathbf{X}) d^{2} \mathbf{x}}_{G_{\text {back }}} & =\omega^{2} \int_{V} \delta v\left(\mathbf{x}^{\prime}\right) G\left(\mathbf{x}^{\prime}, \mathbf{x}_{0}\right)\left[\int_{\partial V} G_{0}\left(\mathbf{x}, \mathbf{x}^{\prime}\right) G_{0}^{*}(\mathbf{x}, \mathbf{X}) d^{2} \mathbf{x}\right] d^{3} \mathbf{x}^{\prime} \\
& \approx \omega^{2} \int_{V} \delta v\left(\mathbf{x}^{\prime}\right) G\left(\mathbf{x}^{\prime}, \mathbf{x}_{0}\right) G_{0}^{*}\left(\mathbf{x}^{\prime}, \mathbf{X}\right) d^{3} \mathbf{x}^{\prime} \\
& +\omega^{2} \int_{V} \delta v\left(\mathbf{x}^{\prime}\right) G\left(\mathbf{x}^{\prime}, \mathbf{x}_{0}\right) G_{0}\left(\mathbf{x}^{\prime}, \mathbf{X}\right) d^{3} \mathbf{x}^{\prime}
\end{aligned}
$$

To get the image, we crosscorrelate $G_{\text {back }}$ with the background Green's function from the surface to the image point, and for the leading-order terms, we replace $G$ by $G_{0}$. Therefore, we get

$$
\begin{aligned}
& \mathbf{I}_{1}(\mathbf{X}) \equiv \int_{\partial V} G_{0}\left(\mathbf{X}, \mathbf{x}_{0}\right) G_{\text {back }}^{*}\left(\mathbf{X}, \mathbf{x}_{0}\right) d^{2} \mathbf{x}_{0} \approx \\
& +\omega^{2} \int_{V} \delta v\left(\mathbf{x}^{\prime}\right) G_{0}\left(\mathbf{x}^{\prime}, \mathbf{X}\right)\left[\int_{\partial V} G_{0}\left(\mathbf{X}, \mathbf{x}_{0}\right) G_{0}^{*}\left(\mathbf{x}_{0}, \mathbf{x}^{\prime}\right) d^{2} \mathbf{x}_{0}\right] d^{3} \mathbf{x}^{\prime} \\
& +\omega^{2} \int_{V} \delta v\left(\mathbf{x}^{\prime}\right) G_{0}^{*}\left(\mathbf{x}^{\prime}, \mathbf{X}\right)\left[\int_{\partial V} G_{0}\left(\mathbf{X}, \mathbf{x}_{0}\right) G_{0}^{*}\left(\mathbf{x}_{0}, \mathbf{x}^{\prime}\right) d^{2} \mathbf{x}_{0}\right] d^{3} \mathbf{x}^{\prime} \\
& \quad+\ldots
\end{aligned}
$$

where $\mathbf{I}_{1}(\mathbf{X})$ is the image of first-order internal multiples (single scattering) in conventional RTM. The surface integral can be performed using equation 2 ; this gives

$$
\begin{aligned}
& \mathbf{I}_{1}(\mathbf{X}) \equiv \int_{\partial V} G_{0}\left(\mathbf{X}, \mathbf{x}_{0}\right) G_{\mathrm{back}}^{*}\left(\mathbf{X}, \mathbf{x}_{0}\right) d^{2} \mathbf{x}_{0} \approx \\
& \omega^{2} \int_{V} \delta v\left(\mathbf{x}^{\prime}\right) G_{0}\left(\mathbf{X}, \mathbf{x}^{\prime}\right) G_{0}^{*}\left(\mathbf{x}^{\prime}, \mathbf{X}\right) d^{3} \mathbf{x}^{\prime} \\
& \quad+\omega^{2} \int_{V} \delta v\left(\mathbf{x}^{\prime}\right) G_{0}\left(\mathbf{X}, \mathbf{x}^{\prime}\right) G_{0}\left(\mathbf{x}^{\prime}, \mathbf{X}\right) d^{3} \mathbf{x}^{\prime} \\
& \quad+\omega^{2} \int_{V} \delta v\left(\mathbf{x}^{\prime}\right) G_{0}^{*}\left(\mathbf{X}, \mathbf{x}^{\prime}\right) G_{0}^{*}\left(\mathbf{x}^{\prime}, \mathbf{X}\right) d^{3} \mathbf{x}^{\prime} \\
& \quad+\omega^{2} \int_{V} \delta v\left(\mathbf{x}^{\prime}\right) G_{0}^{*}\left(\mathbf{X}, \mathbf{x}^{\prime}\right) G_{0}\left(\mathbf{x}^{\prime}, \mathbf{X}\right) d^{3} \mathbf{x}^{\prime} \quad+\ldots
\end{aligned}
$$

The steps described above, namely, back propagation of data, forward propagation of sources, and crosscorrelation, are similar to the conventional RTM approach. The implementation is, however, different. In RTM, usually back and forward propagation are done by solving the wave equation. Here, we use the Green's functions, which are obtained by calculating the traveltime table and then using the traveltimes to get the phase of the Green's function. Amplitudes are left constant; not even the geometric spreading is taken into account. In this case, the back propagation, e.g., would mean correlating the data with the Green's function calculated using traveltimes. Another aspect in which this is equivalent to a conventional RTM with smooth velocity is the absence of reflections because when the migration velocity is smooth, even solving the two-way wave equation does not create reflections. Also because we are calculating the Green's functions and correlating them in the frequency domain, we have no information of time until the last step when we sum over the frequency to apply the zero time imaging condition. This means that in equation 3 (the back propagation step), all times are present, negative and positive. Although we use a Kirchhofftype implementation for back and forward propagation, it is important to note that this is not a requirement. One could solve the wave equation to back propagate the data and forward propagate the source, but this would add to the cost because solving the wave equation is more expensive computationally than the traveltimebased approach.

In equation 5, the right side has four terms. The first two terms are complex conjugates of the last two, and also in the first and fourth terms the $G$ s will give a zero phase for every $\mathbf{X}$. This means that there will be a constant nonzero amplitude everywhere in the image because in that case the sum of these four integrals will just be a real integral with a nonzero (provided there is at least one scatterer in the volume) constant value. This constant amplitude is similar to the low-frequency noise artifact in conventional RTM. By applying a Laplacian filter (Zhang and Sun, 2009), we can get rid of this constant amplitude. Because our examples are 2D, we applied a 2D Laplacian filter with a diagonal $(0,4,0)$. An image is obtained by the fact that when $\mathbf{X} \equiv \mathbf{x}^{\prime}$, all four terms produce a zero phase. For any other $\mathbf{X}$, the second and third terms do not produce a zero phase.

Another point to be noted in equation 5 is that the negative times coming from $G_{\text {back }}$ have not been ignored, as would be done in conventional applications. The negative times result from the first term on the right side of equation 3 . In our case (i.e., working in the frequency domain), ignoring negative times would mean convolving the first term in equation 3 with the Fourier transform of the Heaviside function. To keep things simple, we do not ignore any times in our derivations.

To get the next (higher)-order term, we replace $G$ in $G_{\text {back }}$ with $\int_{V} G_{0}\left(\mathbf{x}, \mathbf{x}^{\prime \prime}\right) \delta v\left(\mathbf{x}^{\prime \prime}\right) G_{0}\left(\mathbf{x}^{\prime \prime}, \mathbf{x}_{0}\right) d^{3} \mathbf{x}^{\prime \prime}$, which is just the Born approximation. Therefore, we get

$$
\begin{aligned}
& \overbrace{\mathbf{I}_{2}(\mathbf{X})}^{\text {conventional }} \equiv \int_{\partial V} G_{0}\left(\mathbf{X}, \mathbf{x}_{0}\right) G_{\text {back }}^{*}\left(\mathbf{X}, \mathbf{x}_{0}\right) d^{2} \mathbf{x}_{0} \approx \\
& +\omega^{2} \int_{V} \delta v\left(\mathbf{x}^{\prime}\right) G_{0}\left(\mathbf{x}^{\prime}, \mathbf{X}\right)\left[\int_{\partial V} G_{0}\left(\mathbf{X}, \mathbf{x}_{0}\right)\left(\int_{V} G_{0}\left(\mathbf{x}^{\prime}, \mathbf{x}^{\prime \prime}\right) \delta v\left(\mathbf{x}^{\prime \prime}\right) G_{0}\left(\mathbf{x}^{\prime \prime}, \mathbf{x}_{0}\right) d^{3} \mathbf{x}^{\prime \prime}\right)^{*} d^{2} \mathbf{x}_{0}\right] d^{3} \mathbf{x}^{\prime} \\
& +\omega^{2} \int_{V} \delta v\left(\mathbf{x}^{\prime}\right) G_{0}^{*}\left(\mathbf{x}^{\prime}, \mathbf{X}\right)\left[\int_{\partial V} G_{0}\left(\mathbf{X}, \mathbf{x}_{0}\right)\left(\int_{V} G_{0}\left(\mathbf{x}^{\prime}, \mathbf{x}^{\prime \prime}\right) \delta v\left(\mathbf{x}^{\prime \prime}\right) G_{0}\left(\mathbf{x}^{\prime \prime}, \mathbf{x}_{0}\right) d^{3} \mathbf{x}^{\prime \prime}\right)^{*} d^{2} \mathbf{x}_{0}\right] d^{3} \mathbf{x}^{\prime} \\
& +\ldots \approx \\
& +\omega^{2} \int_{V} \int_{V} \delta v\left(\mathbf{x}^{\prime}\right) \delta v\left(\mathbf{x}^{\prime \prime}\right) G_{0}\left(\mathbf{x}^{\prime}, \mathbf{X}\right) G_{0}^{*}\left(\mathbf{x}^{\prime}, \mathbf{x}^{\prime \prime}\right)\left[\int_{\partial V} G_{0}\left(\mathbf{X}, \mathbf{x}_{0}\right) G_{0}^{*}\left(\mathbf{x}^{\prime \prime}, \mathbf{x}_{0}\right) d^{2} \mathbf{x}_{0}\right] d^{3} \mathbf{x}^{\prime} d^{3} \mathbf{x}^{\prime \prime} \\
& +\omega^{2} \int_{V} \int_{V} \delta v\left(\mathbf{x}^{\prime}\right) \delta v\left(\mathbf{x}^{\prime \prime}\right) G_{0}^{*}\left(\mathbf{x}^{\prime}, \mathbf{X}\right) G_{0}^{*}\left(\mathbf{x}^{\prime}, \mathbf{x}^{\prime \prime}\right)\left[\int_{\partial V} G_{0}\left(\mathbf{X}, \mathbf{x}_{0}\right) G_{0}^{*}\left(\mathbf{x}^{\prime \prime}, \mathbf{x}_{0}\right) d^{2} \mathbf{x}_{0}\right] d^{3} \mathbf{x}^{\prime} d^{3} \mathbf{x}^{\prime \prime} \\
& +\ldots \approx+\omega^{2} \int_{V} \int_{V} \delta v\left(\mathbf{x}^{\prime}\right) \delta v\left(\mathbf{x}^{\prime \prime}\right) G_{0}\left(\mathbf{x}^{\prime}, \mathbf{X}\right) G_{0}^{*}\left(\mathbf{x}^{\prime}, \mathbf{x}^{\prime \prime}\right) G_{0}\left(\mathbf{x}^{\prime \prime}, \mathbf{X}\right) d^{3} \mathbf{x}^{\prime} d^{3} \mathbf{x}^{\prime \prime} \\
& +\omega_{V} \int_{V} \delta v\left(\mathbf{x}^{\prime}\right) \delta v\left(\mathbf{x}^{\prime \prime}\right) G_{0}\left(\mathbf{x}^{\prime}, \mathbf{X}\right) G_{0}^{*}\left(\mathbf{x}^{\prime}, \mathbf{x}^{\prime \prime}\right) G_{0}^{*}\left(\mathbf{x}^{\prime \prime}, \mathbf{X}\right) d^{3} \mathbf{x}^{\prime} d^{3} \mathbf{x}^{\prime \prime} \\
& +\omega^{2} \int_{V} \int_{V} \delta v\left(\mathbf{x}^{\prime}\right) \delta v\left(\mathbf{x}^{\prime \prime}\right) G_{0}^{*}\left(\mathbf{x}^{\prime}, \mathbf{X}\right) G_{0}^{*}\left(\mathbf{x}^{\prime}, \mathbf{x}^{\prime \prime}\right) G_{0}\left(\mathbf{x}^{\prime \prime}, \mathbf{X}\right) d^{3} \mathbf{x}^{\prime} d^{3} \mathbf{x}^{\prime \prime} \\
& +\omega^{2} \int_{V} \int_{V} \delta v\left(\mathbf{x}^{\prime}\right) \delta v\left(\mathbf{x}^{\prime \prime}\right) G_{0}^{*}\left(\mathbf{x}^{\prime}, \mathbf{X}\right) G_{0}^{*}\left(\mathbf{x}^{\prime}, \mathbf{x}^{\prime \prime}\right) G_{0}^{*}\left(\mathbf{x}^{\prime \prime}, \mathbf{X}\right) d^{3} \mathbf{x}^{\prime} d^{3} \mathbf{x}^{\prime \prime}+\ldots
\end{aligned}
$$

where $\mathbf{I}_{2}^{\mathbf{c}}(\mathbf{X})$ is the image of the second-order Born scattering term (first-order internal multiples) in conventional RTM. From the right side of equation 6 , we see that the zero phase is obtained from the first and the third terms when $\mathbf{X} \equiv \mathbf{x}^{\prime}$ and similarly the first and the second terms give zero phase when $\mathbf{X} \equiv \mathbf{x}^{\prime \prime}$. This means that when the frequencies are summed, i.e., the zero time imaging condition is applied, points $\mathbf{x}^{\prime}$ and $\mathbf{x}^{\prime \prime}$ will be imaged, and they correspond to the scatterers. The fourth term will never contribute 
to the image because the phases of all three Green's functions are added (the total phase will have a minus sign because of the conjugation).

Therefore, from equation 6, we realize that the conventional RTM can also image double scattering, but it will be weaker in amplitude relative to the single-scattering image. This is because the singlescattering image is multiplied by one perturbation term $(\delta v)$, whereas the double-scattering term is multiplied by two perturbation terms. Of course, if the perturbations are large enough, this term would give an image of the double-scattering term, which is higher in amplitude than that of single scattering. In that case, the Born series will not converge and the analysis given here will break down. In practice, the amplitude of the multiple scattering and the perturbation are small and the Born series does converge. Another way to look at the convergence of the scattering series is by analyzing the corresponding series of scattering matrices described by Wapenaar et al. (2010). The scattering matrices approach is applicable here because we have set the amplitude of the Green's functions to be one; i.e., we have regularized them.

In the next section, we describe our three-stage approach to image first-order internal multiples. In the subsequent section, we generalize it to enhance the image of any particular order of internal scattering regardless of the amplitude of perturbation or scattering.

\section{Three-stage procedure}

As discussed above, the second-order term of the Born scattering series in conventional imaging is weaker than the first-order term. This means that we have to either isolate the second-order term or find a series that starts from the second-order term of the Born series so that it is the dominant (subsequent terms have to be weaker to ensure convergence) term. To do that, we suggest a three-stage procedure described as follows:

1) In the first step, we back propagate the recorded data on the surface to obtain $G_{\text {back }}$. This is given by equation 3 .

2) We then crosscorrelate the backward extrapolated recorded data $G_{\text {back }}$ with $G_{s}$ in equation 1 (data recorded on the surface) and get

$$
\begin{aligned}
& \int_{\partial V} G_{s}\left(\mathbf{x}, \mathbf{x}_{0}\right) G_{\text {back }}^{*}\left(\mathbf{X}, \mathbf{x}_{0}\right) d^{2} \mathbf{x}_{0} \approx \\
& \omega^{4} \int_{\partial V}\left[\int_{V} \delta v\left(\mathbf{x}^{\prime \prime}\right) G_{0}\left(\mathbf{x}, \mathbf{x}^{\prime \prime}\right) G\left(\mathbf{x}^{\prime \prime}, \mathbf{x}_{0}\right) d^{3} \mathbf{x}^{\prime \prime}\right] \\
& \times\left[\int_{V} \delta v\left(\mathbf{x}^{\prime}\right) G^{*}\left(\mathbf{x}^{\prime}, \mathbf{x}_{0}\right) G_{0}\left(\mathbf{x}^{\prime}, \mathbf{X}\right) d^{3} \mathbf{x}^{\prime}\right] d^{2} \mathbf{x}_{0} \\
& +\omega^{4} \int_{\partial V}\left[\int_{V} \delta v\left(\mathbf{x}^{\prime \prime}\right) G_{0}\left(\mathbf{x}, \mathbf{x}^{\prime \prime}\right) G\left(\mathbf{x}^{\prime \prime}, \mathbf{x}_{0}\right) d^{3} \mathbf{x}^{\prime \prime}\right] \\
& \times\left[\int_{V} \delta v\left(\mathbf{x}^{\prime}\right) G^{*}\left(\mathbf{x}^{\prime}, \mathbf{x}_{0}\right) G_{0}^{*}\left(\mathbf{x}^{\prime}, \mathbf{X}\right) d^{3} \mathbf{x}^{\prime}\right] d^{2} \mathbf{x}_{0} .
\end{aligned}
$$

$$
\begin{aligned}
& \int_{\partial V} G_{S}\left(\mathbf{x}, \mathbf{x}_{0}\right) G_{\mathrm{back}}^{*}\left(\mathbf{X}, \mathbf{x}_{0}\right) d^{2} \mathbf{x}_{0} \approx \\
& \omega^{4} \int_{\partial V}\left[\int_{V} \delta v\left(\mathbf{x}^{\prime \prime}\right) G_{0}\left(\mathbf{x}, \mathbf{x}^{\prime \prime}\right) G_{0}\left(\mathbf{x}^{\prime \prime}, \mathbf{x}_{0}\right) d^{3} \mathbf{x}^{\prime \prime}\right] \\
& \times\left[\int_{V} \delta v\left(\mathbf{x}^{\prime}\right) G_{0}^{*}\left(\mathbf{x}^{\prime}, \mathbf{x}_{0}\right) G_{0}\left(\mathbf{x}^{\prime}, \mathbf{X}\right) d^{3} \mathbf{x}^{\prime}\right] d^{2} \mathbf{x}_{0} \\
& +\omega^{4} \int_{\partial V}\left[\int_{V} \delta v\left(\mathbf{x}^{\prime \prime}\right) G_{0}\left(\mathbf{x}, \mathbf{x}^{\prime \prime}\right) G_{0}\left(\mathbf{x}^{\prime \prime}, \mathbf{x}_{0}\right) d^{3} \mathbf{x}^{\prime \prime}\right] \\
& \times\left[\int_{V} \delta v\left(\mathbf{x}^{\prime}\right) G_{0}^{*}\left(\mathbf{x}^{\prime}, \mathbf{x}_{0}\right) G_{0}^{*}\left(\mathbf{x}^{\prime}, \mathbf{X}\right) d^{3} \mathbf{x}^{\prime}\right] d^{2} \mathbf{x}_{0} \\
& +\ldots
\end{aligned}
$$

Then, by rearranging the integrals in equation 8 and using equation 2 to perform the surface integral, we get

$$
\begin{aligned}
& \int_{\partial V} G_{s}\left(\mathbf{x}, \mathbf{x}_{0}\right) G_{\text {back }}^{*}\left(\mathbf{X}, \mathbf{x}_{0}\right) d^{2} \mathbf{x}_{0} \approx \\
& \omega^{4} \overbrace{\int_{V} \int_{V} \delta v\left(\mathbf{x}^{\prime}\right) \delta v\left(\mathbf{x}^{\prime \prime}\right) G_{0}\left(\mathbf{x}, \mathbf{x}^{\prime \prime}\right) G_{0}\left(\mathbf{x}^{\prime \prime}, \mathbf{x}^{\prime}\right) G_{0}\left(\mathbf{x}^{\prime}, \mathbf{X}\right) d^{3} \mathbf{x}^{\prime} d^{3} \mathbf{x}^{\prime \prime}}^{\text {second-order term of the Born scattering series }} \\
& +\omega^{4} \int_{V} \int_{V} \delta v\left(\mathbf{x}^{\prime}\right) \delta v\left(\mathbf{x}^{\prime \prime}\right) G_{0}\left(\mathbf{x}, \mathbf{x}^{\prime \prime}\right) G_{0}^{*}\left(\mathbf{x}^{\prime \prime}, \mathbf{x}^{\prime}\right) G_{0}\left(\mathbf{x}^{\prime}, \mathbf{X}\right) d^{3} \mathbf{x}^{\prime} d^{3} \mathbf{x}^{\prime \prime} \\
& +\omega^{4} \int_{V} \int_{V} \delta v\left(\mathbf{x}^{\prime}\right) \delta v\left(\mathbf{x}^{\prime \prime}\right) G_{0}\left(\mathbf{x}, \mathbf{x}^{\prime \prime}\right) G_{0}\left(\mathbf{x}^{\prime \prime}, \mathbf{x}^{\prime}\right) G_{0}^{*}\left(\mathbf{x}^{\prime}, \mathbf{X}\right) d^{3} \mathbf{x}^{\prime} d^{3} \mathbf{x}^{\prime \prime} \\
& +\omega^{4} \int_{V} \int_{V} \delta v\left(\mathbf{x}^{\prime}\right) \delta v\left(\mathbf{x}^{\prime \prime}\right) G_{0}\left(\mathbf{x}, \mathbf{x}^{\prime \prime}\right) G_{0}^{*}\left(\mathbf{x}^{\prime \prime}, \mathbf{x}^{\prime}\right) G_{0}^{*}\left(\mathbf{x}^{\prime}, \mathbf{X}\right) d^{3} \mathbf{x}^{\prime} d^{3} \mathbf{x}^{\prime \prime} \\
& +\ldots
\end{aligned}
$$

We can already see that by crosscorrelating the back-propagated recorded data from stage one, with the recorded data on the surface, we extract the first-order internal scattering. In this process, note that the first-order internal multiples were obtained using only the background Green's function.

3) Finally, we get to the imaging step. We crosscorrelate equation 9 with the background Green's function from surface to $\mathbf{X}$ :

$$
\begin{aligned}
& \mathbf{I}_{2}(\mathbf{X}) \equiv \int_{\partial V} G_{0}(\mathbf{X}, \mathbf{x})\left[\int_{\partial V} G_{s}\left(\mathbf{x}, \mathbf{x}_{0}\right) G_{\mathrm{back}}^{*}\left(\mathbf{X}, \mathbf{x}_{0}\right) d^{2} \mathbf{x}_{0}\right]^{*} d^{2} \mathbf{x} \approx \\
& \omega^{4} \int_{\partial V} G_{0}(\mathbf{X}, \mathbf{x})\left[\int_{V} \int_{V} \delta v\left(\mathbf{x}^{\prime}\right) \delta v\left(\mathbf{x}^{\prime \prime}\right) G_{0}\left(\mathbf{x}, \mathbf{x}^{\prime \prime}\right) G_{0}\left(\mathbf{x}^{\prime \prime}, \mathbf{x}^{\prime}\right) G_{0}\left(\mathbf{x}^{\prime}, \mathbf{X}\right) d^{3} \mathbf{x}^{\prime} d^{3} \mathbf{x}^{\prime \prime}\right]^{*} d^{2} \mathbf{x} \\
& +\omega^{4} \int_{\partial V} G_{0}(\mathbf{X}, \mathbf{x})\left[\int_{V} \int_{V} \delta v\left(\mathbf{x}^{\prime}\right) \delta v\left(\mathbf{x}^{\prime \prime}\right) G_{0}\left(\mathbf{x}, \mathbf{x}^{\prime \prime}\right) G_{0}^{*}\left(\mathbf{x}^{\prime \prime}, \mathbf{x}^{\prime}\right) G_{0}\left(\mathbf{x}^{\prime}, \mathbf{X}\right) d^{3} \mathbf{x}^{\prime} d^{3} \mathbf{x}^{\prime \prime}\right]^{*} d^{2} \mathbf{x} \\
& +\omega^{4} \int_{\partial V} G_{0}(\mathbf{X}, \mathbf{x})\left[\int_{V} \int_{V} \delta v\left(\mathbf{x}^{\prime}\right) \delta v\left(\mathbf{x}^{\prime \prime}\right) G_{0}\left(\mathbf{x}, \mathbf{x}^{\prime \prime}\right) G_{0}\left(\mathbf{x}^{\prime \prime}, \mathbf{x}^{\prime}\right) G_{0}^{*}\left(\mathbf{x}^{\prime}, \mathbf{X}\right) d^{3} \mathbf{x}^{\prime} d^{3} \mathbf{x}^{\prime \prime}\right]^{*} d^{2} \mathbf{x} \\
& +\omega^{4} \int_{\partial V} G_{0}(\mathbf{X}, \mathbf{x})\left[\int_{V} \int_{V} \delta v\left(\mathbf{x}^{\prime}\right) \delta v\left(\mathbf{x}^{\prime \prime}\right) G_{0}\left(\mathbf{x}, \mathbf{x}^{\prime \prime}\right) G_{0}^{*}\left(\mathbf{x}^{\prime \prime}, \mathbf{x}^{\prime}\right) G_{0}^{*}\left(\mathbf{x}^{\prime}, \mathbf{X}\right) d^{3} \mathbf{x}^{\prime} d^{3} \mathbf{x}^{\prime \prime}\right]^{*} d^{2} \mathbf{x} \\
& +\ldots
\end{aligned}
$$

By considering $G\left(\mathbf{x}^{\prime}, \mathbf{x}^{\prime \prime}\right) \equiv G_{0}\left(\mathbf{x}^{\prime}, \mathbf{x}^{\prime \prime}\right)+G_{s}\left(\mathbf{x}^{\prime}, \mathbf{x}^{\prime \prime}\right)$, the leading-order terms on the right side of equation 7 are
Again, using equation 2, we get eight leading-order terms, i.e., 


$$
\begin{aligned}
& \int_{\partial V} G_{0}(\mathbf{X}, \mathbf{x})\left[\int_{\partial V} G_{s}\left(\mathbf{x}, \mathbf{x}_{0}\right) G_{\mathrm{back}}^{*}\left(\mathbf{X}, \mathbf{x}_{0}\right) d^{2} \mathbf{x}_{0}\right]^{*} d^{2} \mathbf{x} \approx \\
& \omega^{4} \int_{V} \int_{V} \delta v\left(\mathbf{x}^{\prime}\right) \delta v\left(\mathbf{x}^{\prime \prime}\right) G_{0}\left(\mathbf{X}, \mathbf{x}^{\prime \prime}\right) G_{0}^{*}\left(\mathbf{x}^{\prime \prime}, \mathbf{x}^{\prime}\right) G_{0}^{*}\left(\mathbf{x}^{\prime}, \mathbf{X}\right) d^{3} \mathbf{x}^{\prime} d^{3} \mathbf{x}^{\prime \prime} \\
& +\omega^{4} \int_{V} \int_{V} \delta v\left(\mathbf{x}^{\prime}\right) \delta v\left(\mathbf{x}^{\prime \prime}\right) G_{0}\left(\mathbf{X}, \mathbf{x}^{\prime \prime}\right) G_{0}\left(\mathbf{x}^{\prime \prime}, \mathbf{x}^{\prime}\right) G_{0}^{*}\left(\mathbf{x}^{\prime}, \mathbf{X}\right) d^{3} \mathbf{x}^{\prime} d^{3} \mathbf{x}^{\prime \prime} \\
& +\omega^{4} \int_{V} \int_{V} \delta v\left(\mathbf{x}^{\prime}\right) \delta v\left(\mathbf{x}^{\prime \prime}\right) G_{0}\left(\mathbf{X}, \mathbf{x}^{\prime \prime}\right) G_{0}^{*}\left(\mathbf{x}^{\prime \prime}, \mathbf{x}^{\prime}\right) G_{0}\left(\mathbf{x}^{\prime}, \mathbf{X}\right) d^{3} \mathbf{x}^{\prime} d^{3} \mathbf{x}^{\prime \prime} \\
& +\omega^{4} \int_{V} \int_{V} \delta v\left(\mathbf{x}^{\prime}\right) \delta v\left(\mathbf{x}^{\prime \prime}\right) G_{0}\left(\mathbf{X}, \mathbf{x}^{\prime \prime}\right) G_{0}\left(\mathbf{x}^{\prime \prime}, \mathbf{x}^{\prime}\right) G_{0}\left(\mathbf{x}^{\prime}, \mathbf{X}\right) d^{3} \mathbf{x}^{\prime} d^{3} \mathbf{x}^{\prime \prime} \\
& +\omega^{4} \int_{V} \int_{V} \delta v\left(\mathbf{x}^{\prime}\right) \delta v\left(\mathbf{x}^{\prime \prime}\right) G_{0}^{*}\left(\mathbf{X}, \mathbf{x}^{\prime \prime}\right) G_{0}^{*}\left(\mathbf{x}^{\prime \prime}, \mathbf{x}^{\prime}\right) G_{0}^{*}\left(\mathbf{x}^{\prime}, \mathbf{X}\right) d^{3} \mathbf{x}^{\prime} d^{3} \mathbf{x}^{\prime \prime} \\
& +\omega^{4} \int_{V} \int_{V} \delta v\left(\mathbf{x}^{\prime}\right) \delta v\left(\mathbf{x}^{\prime \prime}\right) G_{0}^{*}\left(\mathbf{X}, \mathbf{x}^{\prime \prime}\right) G_{0}\left(\mathbf{x}^{\prime \prime}, \mathbf{x}^{\prime}\right) G_{0}^{*}\left(\mathbf{x}^{\prime}, \mathbf{X}\right) d^{3} \mathbf{x}^{\prime} d^{3} \mathbf{x}^{\prime \prime} \\
& +\omega^{4} \int_{V} \int_{V} \delta v\left(\mathbf{x}^{\prime}\right) \delta v\left(\mathbf{x}^{\prime \prime}\right) G_{0}^{*}\left(\mathbf{X}, \mathbf{x}^{\prime \prime}\right) G_{0}^{*}\left(\mathbf{x}^{\prime \prime}, \mathbf{x}^{\prime}\right) G_{0}\left(\mathbf{x}^{\prime}, \mathbf{X}\right) d^{3} \mathbf{x}^{\prime} d^{3} \mathbf{x}^{\prime \prime} \\
& +\omega^{4} \int_{V} \delta v\left(\mathbf{x}_{V}^{\prime}\right) \delta v\left(\mathbf{x}^{\prime \prime}\right) G_{0}^{*}\left(\mathbf{X}, \mathbf{x}^{\prime \prime}\right) G_{0}\left(\mathbf{x}^{\prime \prime}, \mathbf{x}^{\prime}\right) G_{0}\left(\mathbf{x}^{\prime}, \mathbf{X}\right) d^{3} \mathbf{x}^{\prime} d^{3} \mathbf{x}^{\prime \prime} \\
& +\ldots
\end{aligned}
$$

From the second, third, sixth, and seventh terms on the right side of equation 11 , we observe that when the image point $\mathbf{X} \equiv \mathbf{x}^{\prime \prime}$, we get a zero phase. For example, in the term

$$
\omega^{4} \int_{V} \delta v\left(\mathbf{x}^{\prime}\right) \delta v\left(\mathbf{x}^{\prime \prime}\right) \underbrace{G_{0}\left(\mathbf{x}^{\prime \prime}, \mathbf{x}^{\prime \prime}\right)}_{\text {zero phase }} \underbrace{G_{0}\left(\mathbf{x}^{\prime \prime}, \mathbf{x}^{\prime}\right) G_{0}^{*}\left(\mathbf{x}^{\prime}, \mathbf{x}^{\prime \prime}\right)}_{\text {zero phase }} d^{3} \mathbf{x}^{\prime} d^{3} \mathbf{x}^{\prime \prime},
$$

the Green's functions have zero phase as indicated. Thus, when we apply the zero-time imaging condition (i.e., summation over frequencies) this term will contribute to the image. Similarly for the first, third, sixth, and eighth terms will give zero phase when $\mathbf{X} \equiv \mathbf{x}^{\prime}$. For example, in the term

$$
\omega^{4} \int_{V} \delta v\left(\mathbf{x}^{\prime}\right) \delta v\left(\mathbf{x}^{\prime \prime}\right) \underbrace{G_{0}\left(\mathbf{x}^{\prime}, \mathbf{x}^{\prime \prime}\right) G_{0}^{*}\left(\mathbf{x}^{\prime \prime}, \mathbf{x}^{\prime}\right)}_{\text {zero phase }} \underbrace{G_{0}^{*}\left(\mathbf{x}^{\prime}, \mathbf{x}^{\prime}\right)}_{\text {zero phase }} d^{3} \mathbf{x}^{\prime} d^{3} \mathbf{x}^{\prime \prime},
$$

the Green's functions have zero phase as indicated. The fourth and fifth terms in equation 11 will never give a zero phase and thus, will never contribute to the image. Also, note that the first four terms on the right side of equation 11 are the complex conjugate of the last four, this would give a constant amplitude all over the image, which can be removed by applying the Laplacian filter.

\section{Generalized internal multiple imaging}

It is straightforward to generalize the three-stage procedure to image higher order internal multiples. To do that, we just have to repeat stage two of the above procedure multiples, $N-1$ times in total, where $N$ is the order of terms in the Born scattering series we desire to image. For the third-order term (second-order internal multiples), this can be written as

$$
\begin{aligned}
& \mathbf{I}_{3} \equiv \int_{\partial V} G_{0}(\mathbf{X}, \mathbf{x})\left[\int_{\partial V} G_{s}\left(\mathbf{x}, \mathbf{x}_{1}\right)\left[\int_{\partial V} G_{s}\left(\mathbf{x}_{1}, \mathbf{x}_{0}\right) G_{\text {back }}^{*}\left(\mathbf{X}, \mathbf{x}_{0}\right) d^{2} \mathbf{x}_{0}\right]^{*} d^{2} \mathbf{x}_{1}\right]^{*} d^{2} \mathbf{x} \approx \\
& \omega^{6} \int_{V} \int_{V} \int_{V} \delta v\left(\mathbf{x}^{\prime}\right) \delta v\left(\mathbf{x}^{\prime \prime}\right) \delta v\left(\mathbf{x}^{\prime \prime \prime}\right) G_{0}\left(\mathbf{X}, \mathbf{x}^{\prime \prime \prime}\right) G_{0}^{*}\left(\mathbf{x}^{\prime \prime \prime}, \mathbf{x}^{\prime \prime}\right) G_{0}\left(\mathbf{x}^{\prime \prime}, \mathbf{x}^{\prime}\right) \\
& \times G_{0}\left(\mathbf{x}^{\prime}, \mathbf{X}\right) d^{3} \mathbf{x}^{\prime} d^{3} \mathbf{x}^{\prime \prime} d^{3} \mathbf{x}^{\prime \prime \prime} \\
& +\omega^{6} \int_{V} \int_{V} \int_{V} \delta v\left(\mathbf{x}^{\prime}\right) \delta v\left(\mathbf{x}^{\prime \prime}\right) \delta v\left(\mathbf{x}^{\prime \prime \prime}\right) G_{0}\left(\mathbf{X}, \mathbf{x}^{\prime \prime \prime}\right) G_{0}^{*}\left(\mathbf{x}^{\prime \prime \prime}, \mathbf{x}^{\prime \prime}\right) G_{0}\left(\mathbf{x}^{\prime \prime}, \mathbf{x}^{\prime}\right) \\
& \times G_{0}^{*}\left(\mathbf{x}^{\prime}, \mathbf{X}\right) d^{3} \mathbf{x}^{\prime} d^{3} \mathbf{x}^{\prime \prime} d^{3} \mathbf{x}^{\prime \prime \prime} \\
& +\ldots
\end{aligned}
$$

Only the first two terms are shown in equation 14 (all sixteen terms for imaging the second-order internal multiples together with the derivation are given in Appendix A). The imaging terms on the right side of equation 14 (equation A-3) can be analyzed in the same way as equation 11. For example, from the second term on the right side of equation 14, we can see that when the image point $\mathbf{X} \equiv \mathbf{x}^{\prime \prime}$, this term has a zero phase and thus, this point will be imaged. Also, the first term on the right side of equation 14 does not give a zero phase no matter where $\mathbf{X}$ is and, therefore, it does not contribute to the image.

\section{Crosstalk suppression}

The image I resulting from the additional crosscorrelation will include crosstalk terms because the Green's functions used in the imaging terms might result in a total phase of any term to be zero even when the imaging point does not correspond to a scatterer position. However, all the contributing terms will not have zero phase at the wrong point simultaneously, and thus, their amplitude is expected to be weaker than the image of the scatterers. Based on this assumption, we use a simple procedure to eliminate the crosstalk, given by squaring the image. The squaring of the image, however, can also result from an optimization approach, in which the objective function is the dot product (crosscorrelation) of the modeled and the recorded data.

The crosstalk of these multiples is also present in the singlescattering image, as well. Wong et al. (2012) suggest a method to suppress such noise. However, it will require a least-squares formulation of the imaging process, in which we fit the modeled data to the recorded data. Because the fitting process requires that the amplitudes be accurate, we alternatively rely on a dot product (zero-lag crosscorrelation) of the modeled and recorded surface data. In this case, the initial image is used to model the scatterers (Wong et al., 2011 , 2012). For double scattering, the initial image $\left(\mathbf{I}_{2}\right)$ is multiplied by the back-propagated wavefield, convolved again with the background Green's function to the surface, and crosscorrelated with the recorded surface data, as follows:

$$
\begin{aligned}
& \int_{\partial V} \int_{\partial V} G_{S}\left(\mathbf{x}, \mathbf{x}_{0}\right)\left[G_{\text {back }}\left(\mathbf{x}_{0}, \mathbf{X}\right) G_{0}(\mathbf{x}, \mathbf{X}) \mathbf{I}_{2}(\mathbf{X})\right]^{*} d^{2} \mathbf{x}_{0} d^{2} \mathbf{x} \\
& =\mathbf{I}_{2}(\mathbf{X}) \omega^{4} \int_{\partial V} \int_{\partial V}\left[\int_{V} \int_{V} \delta v\left(\mathbf{x}^{\prime}\right) \delta v\left(\mathbf{x}^{\prime \prime}\right) G\left(\mathbf{x}^{\prime \prime}, \mathbf{x}_{0}\right)\right. \\
& \left.\times G_{0}\left(\mathbf{x}, \mathbf{x}^{\prime \prime}\right) G^{*}\left(\mathbf{x}^{\prime}, \mathbf{x}_{0}\right) G_{0}\left(\mathbf{x}^{\prime}, \mathbf{X}\right) G_{0}^{*}(\mathbf{x}, \mathbf{X}) d^{3} \mathbf{x}^{\prime} d^{3} \mathbf{x}^{\prime \prime}\right] d^{2} \mathbf{x}_{0} d^{2} \mathbf{x} \\
& +\mathbf{I}_{2}(\mathbf{X}) \omega^{4} \int_{\partial V} \int_{\partial V}\left[\int_{V} \int_{V} \delta v\left(\mathbf{x}^{\prime}\right) \delta v\left(\mathbf{x}^{\prime \prime}\right) G\left(\mathbf{x}^{\prime \prime}, \mathbf{x}_{0}\right)\right. \\
& \left.\times G_{0}\left(\mathbf{x}, \mathbf{x}^{\prime \prime}\right) G^{*}\left(\mathbf{x}^{\prime}, \mathbf{x}_{0}\right) G_{0}^{*}\left(\mathbf{x}^{\prime}, \mathbf{X}\right) G_{0}^{*}(\mathbf{x}, \mathbf{X}) d^{3} \mathbf{x}^{\prime} d^{3} \mathbf{x}^{\prime \prime}\right] d^{2} \mathbf{x}_{0} d^{2} \mathbf{x} .
\end{aligned}
$$


Then, we rearrange the integrals and consider the leading-order terms, i.e.,

$$
\begin{aligned}
& \int_{\partial V} \int_{\partial V} G_{S}\left(\mathbf{x}, \mathbf{x}_{0}\right)\left[G_{\text {back }}\left(\mathbf{x}_{0}, \mathbf{X}\right) G_{0}(\mathbf{x}, \mathbf{X}) \mathbf{I}_{2}(\mathbf{X})\right]^{*} d^{2} \mathbf{x}_{0} d^{2} \mathbf{x} \\
& =\mathbf{I}_{2}(\mathbf{X}) \omega^{4} \int_{V} \int_{V} \delta v\left(\mathbf{x}^{\prime}\right) \delta v\left(\mathbf{x}^{\prime \prime}\right)\left[\int_{\partial V} \int_{\partial V} G_{0}\left(\mathbf{x}^{\prime \prime}, \mathbf{X}\right) G_{0}\left(\mathbf{x}^{\prime}, \mathbf{x}_{0}\right)\right. \\
& \left.\times G_{0}^{*}\left(\mathbf{x}^{\prime \prime}, \mathbf{x}_{0}\right) G_{0}\left(\mathbf{x}, \mathbf{x}^{\prime}\right) G_{0}^{*}(\mathbf{x}, \mathbf{X}) d^{2} \mathbf{x}_{0} d^{2} \mathbf{x}\right] d^{3} \mathbf{x}^{\prime} d^{3} \mathbf{x}^{\prime \prime} \\
& +\mathbf{I}_{2}(\mathbf{X}) \omega^{4} \int_{V} \int_{V} \delta v\left(\mathbf{x}^{\prime}\right) \delta v\left(\mathbf{x}^{\prime \prime}\right)\left[\int_{\partial V} \int_{\partial V} G_{0}^{*}\left(\mathbf{x}^{\prime \prime}, \mathbf{X}\right) G_{0}\left(\mathbf{x}^{\prime}, \mathbf{x}_{0}\right)\right. \\
& \left.\times G_{0}^{*}\left(\mathbf{x}^{\prime \prime}, \mathbf{x}_{0}\right) G_{0}\left(\mathbf{x}, \mathbf{x}^{\prime}\right) G_{0}^{*}(\mathbf{x}, \mathbf{X}) d^{2} \mathbf{x}_{0} d^{2} \mathbf{x}\right] d^{3} \mathbf{x}^{\prime} d^{3} \mathbf{x}^{\prime \prime} \\
& +\ldots
\end{aligned}
$$

Using equation 2 , we get

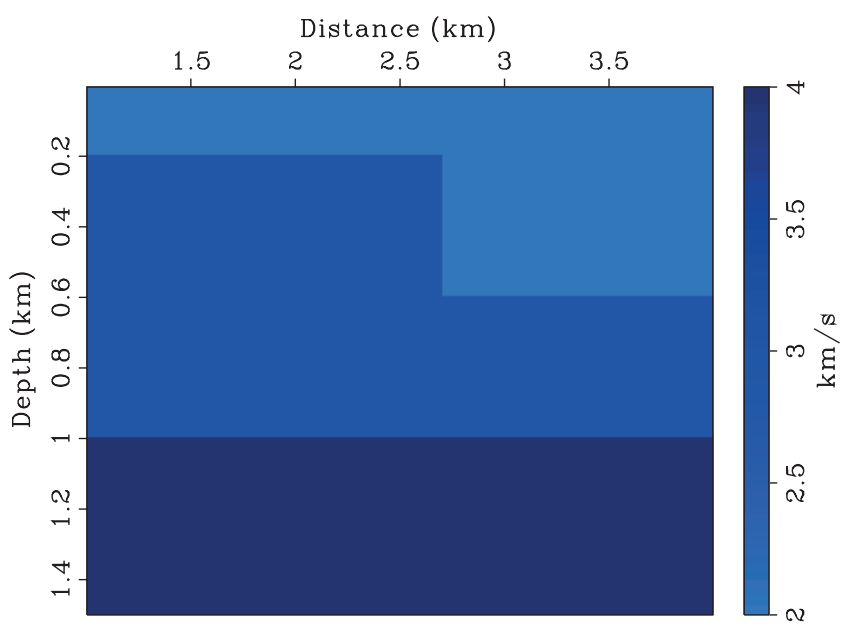

Figure 1. Velocity model.

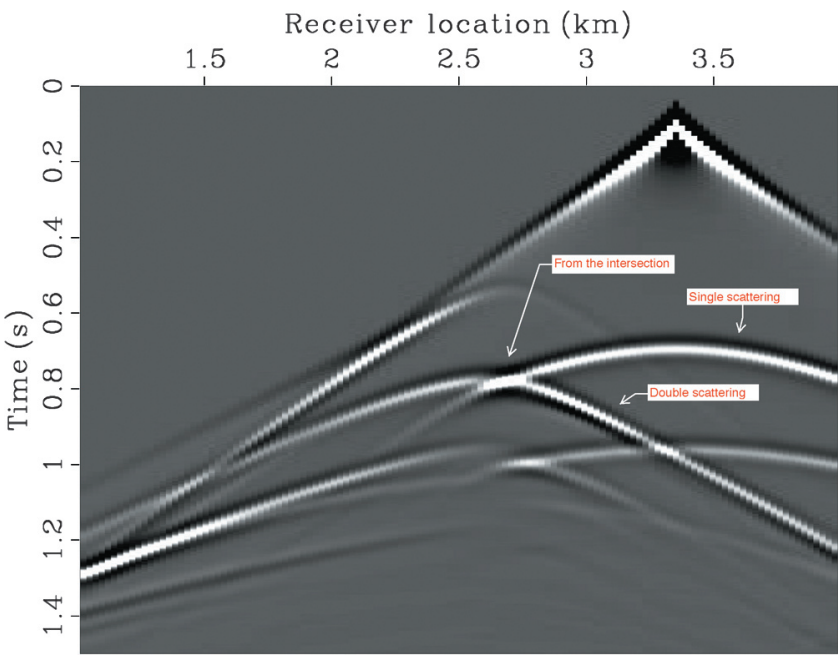

Figure 2. Source on the surface at horizontal location $3.35 \mathrm{~km}$.

$$
\begin{aligned}
& \int_{\partial V} \int_{\partial V} G_{S}\left(\mathbf{x}, \mathbf{x}_{0}\right)\left[G_{\mathrm{back}}\left(\mathbf{x}_{0}, \mathbf{X}\right) G_{0}(\mathbf{x}, \mathbf{X}) \mathbf{I}_{2}(\mathbf{X})\right]^{*} d^{2} \mathbf{x}_{0} d^{2} \mathbf{x} \approx \\
& \mathbf{I}_{2}\left[\omega^{4} \int_{V} \int_{V} \delta v\left(\mathbf{x}^{\prime}\right) \delta v\left(\mathbf{x}^{\prime \prime}\right) G_{0}\left(\mathbf{x}^{\prime \prime}, \mathbf{X}\right) G_{0}\left(\mathbf{x}^{\prime \prime}, \mathbf{x}^{\prime}\right) G_{0}^{*}\left(\mathbf{x}^{\prime}, \mathbf{X}\right) d^{3} \mathbf{x}^{\prime} d^{3} \mathbf{x}^{\prime \prime}\right. \\
& +\omega^{4} \int_{V} \int_{V} \delta v\left(\mathbf{x}^{\prime}\right) \delta v\left(\mathbf{x}^{\prime \prime}\right) G_{0}\left(\mathbf{x}^{\prime \prime}, \mathbf{X}\right) G_{0}^{*}\left(\mathbf{x}^{\prime \prime}, \mathbf{x}^{\prime}\right) G_{0}^{*}\left(\mathbf{x}^{\prime}, \mathbf{X}\right) d^{3} \mathbf{x}^{\prime} d^{3} \mathbf{x}^{\prime \prime} \\
& +\omega^{4} \int_{V} \int_{V} \delta v\left(\mathbf{x}^{\prime}\right) \delta v\left(\mathbf{x}^{\prime \prime}\right) G_{0}\left(\mathbf{x}^{\prime \prime}, \mathbf{X}\right) G_{0}\left(\mathbf{x}^{\prime \prime}, \mathbf{x}^{\prime}\right) G_{0}\left(\mathbf{x}^{\prime}, \mathbf{X}\right) d^{3} \mathbf{x}^{\prime} d^{3} \mathbf{x}^{\prime \prime} \\
& +\omega^{4} \int_{V} \int_{V} \delta v\left(\mathbf{x}^{\prime}\right) \delta v\left(\mathbf{x}^{\prime \prime}\right) G_{0}\left(\mathbf{x}^{\prime \prime}, \mathbf{X}\right) G_{0}^{*}\left(\mathbf{x}^{\prime \prime}, \mathbf{x}^{\prime}\right) G_{0}\left(\mathbf{x}^{\prime}, \mathbf{X}\right) d^{3} \mathbf{x}^{\prime} d^{3} \mathbf{x}^{\prime \prime} \\
& +\omega^{4} \int_{V} \int_{V} \delta v\left(\mathbf{x}^{\prime}\right) \delta v\left(\mathbf{x}^{\prime \prime}\right) G_{0}^{*}\left(\mathbf{x}^{\prime \prime}, \mathbf{X}\right) G_{0}\left(\mathbf{x}^{\prime \prime}, \mathbf{x}^{\prime}\right) G_{0}^{*}\left(\mathbf{x}^{\prime}, \mathbf{X}\right) d^{3} \mathbf{x}^{\prime} d^{3} \mathbf{x}^{\prime \prime} \\
& +\omega^{4} \int_{V} \int_{V} \delta v\left(\mathbf{x}^{\prime}\right) \delta v\left(\mathbf{x}^{\prime \prime}\right) G_{0}^{*}\left(\mathbf{x}^{\prime \prime}, \mathbf{X}\right) G_{0}^{*}\left(\mathbf{x}^{\prime \prime}, \mathbf{x}^{\prime}\right) G_{0}^{*}\left(\mathbf{x}^{\prime}, \mathbf{X}\right) d^{3} \mathbf{x}^{\prime} d^{3} \mathbf{x}^{\prime \prime} \\
& +\omega^{4} \int_{V} \int_{V} \delta v\left(\mathbf{x}^{\prime}\right) \delta v\left(\mathbf{x}^{\prime \prime}\right) G_{0}^{*}\left(\mathbf{x}^{\prime \prime}, \mathbf{X}\right) G_{0}\left(\mathbf{x}^{\prime \prime}, \mathbf{x}^{\prime}\right) G_{0}\left(\mathbf{x}^{\prime}, \mathbf{X}\right) d^{3} \mathbf{x}^{\prime} d^{3} \mathbf{x}^{\prime \prime} \\
& +\omega^{4} \int_{V} \int_{V} \delta v\left(\mathbf{x}^{\prime}\right) \delta v\left(\mathbf{x}^{\prime \prime}\right) G_{0}^{*}\left(\mathbf{x}^{\prime \prime}, \mathbf{X}\right) G_{0}^{*}\left(\mathbf{x}^{\prime \prime}, \mathbf{x}^{\prime}\right) G_{0}\left(\mathbf{x}^{\prime}, \mathbf{X}\right) d^{3} \mathbf{x}^{\prime} d^{3} \mathbf{x}^{\prime \prime} \\
& +\ldots]=(\mathbf{I})^{2} .
\end{aligned}
$$

Equation 17 shows that the crosstalk suppression method described here is just squaring the image.

\section{Examples}

We apply this approach first on a simple model shown in Figure 1. In this velocity model, the vertical reflector at the horizontal location just before $3 \mathrm{~km}$ will not be illuminated by the single-scattering recorded energy on the surface. It, however, will be illuminated by a double bounce using the horizontal reflector at a depth of $0.6 \mathrm{~km}$. To image these first-order internal multiples (second-order term in the Born series), we generate synthetic seismic data using a finite-difference scheme (fourth order in space and second order in time) for modeling an acoustic wave. Sources and receivers cover the whole surface with a spacing of $24 \mathrm{~m}$. Figure 2 shows a shot gather with the source at $3.35 \mathrm{~km}$. The single-scattering labeled in Figure 2 is a reflection from the horizontal layer and a depth of $0.6 \mathrm{~km}$, and the double-scattering event shown is the wave, which bounced first from the horizontal reflector and then the vertical one. The singleand double-scattering events converge at a point labeled from the intersection, where the intersection is between the vertical reflector and the horizontal one at a $0.6 \mathrm{~km}$ depth. To implement the migration algorithm, first traveltimes are calculated from each source/ receiver position on the surface to every point in the model. These traveltimes are then used to calculate the phase of the background Green's function. This background Green's function is crosscorrelated with the data; this is back propagation. For single scattering, we crosscorrelate this back-propagated wavefield with the background Green's function. For double scattering, as described in the theory, we crosscorrelate the back-propagated wavefield with the data and then crosscorrelate with surface to image point background Green's function. With this implementation scheme, we apply GIMI for first-order internal multiples. Indeed, in Figure 3, we see that the vertical reflector has extremely low amplitude relative to the horizontal reflectors. However, when we image the secondorder term, we see in Figure 4 that this reflector is clearly visible. 
The summed image in Figure 5 shows all the reflectors. The images in Figures 3-5 have crosstalk noise in them. To suppress such noise, we use the dot product approach described earlier. Figures $6-8$ show the images after the crosstalk suppression. The image is reasonably cleaner.

Finally, we apply our method on a more complicated model, given by a portion from the Marmousi velocity model shown in Figure 9. A shot gather at $1 \mathrm{~km}$ is shown in Figure 10. Figures 1113 show the images of the model in Figure 9. We show only the images obtained after crosstalk suppression. Figures 11-13 show the image points, which took part in the double scattering. In Figure 13, the faults that have been resolved much more clearly that the single-scattering image in Figure 11. These are not the only points involved in multiple scattering, but they are the most prominent ones because the velocity contrast was high.

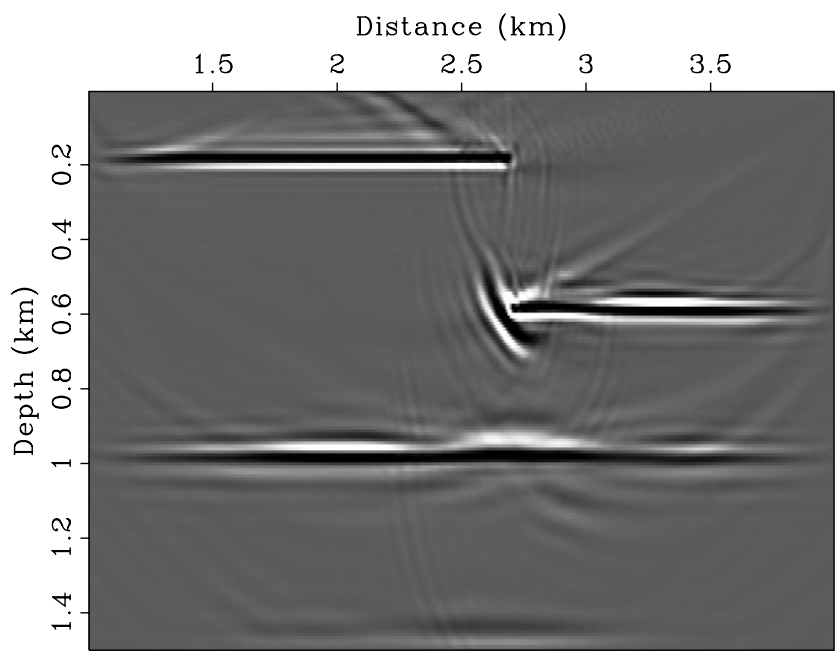

Figure 3. Single scattering image. With (multiple-induced) crosstalk.

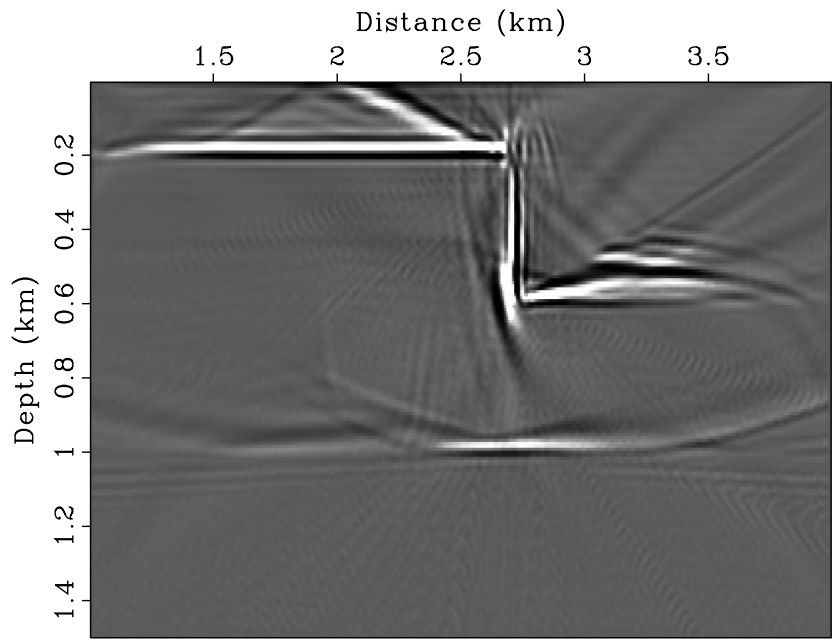

Figure 4. Image using the second-order Born scattering term. With (multiple-induced) crosstalk.

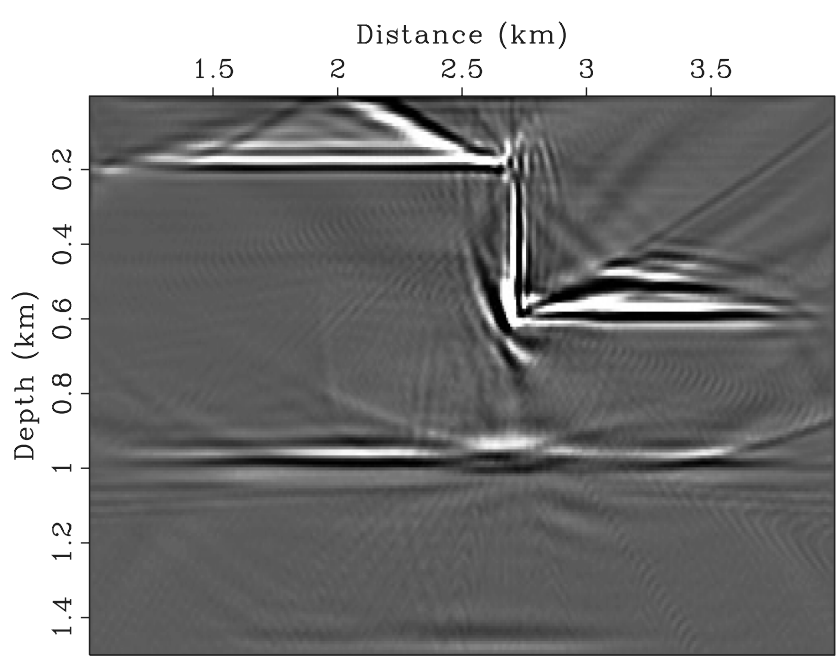

Figure 5. Sum of images in Figures 3 and 4.

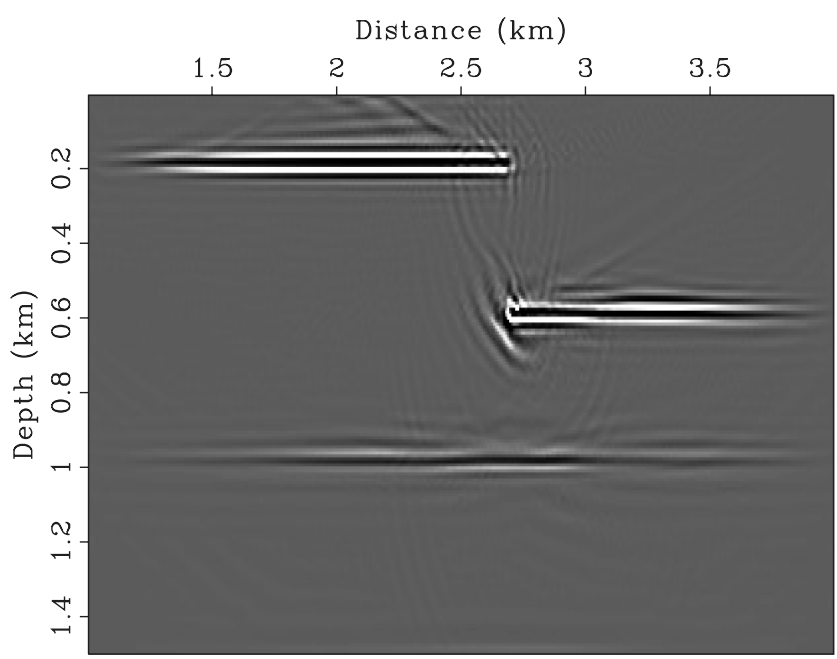

Figure 6. Single scattering image. After crosstalk suppression.

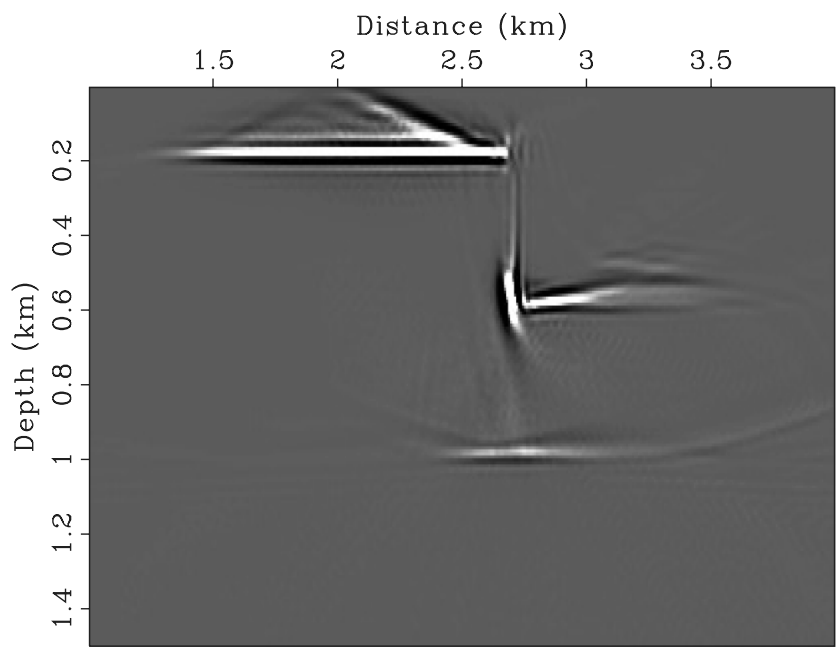

Figure 7. Image using the second-order Born scattering term. After crosstalk suppression. 


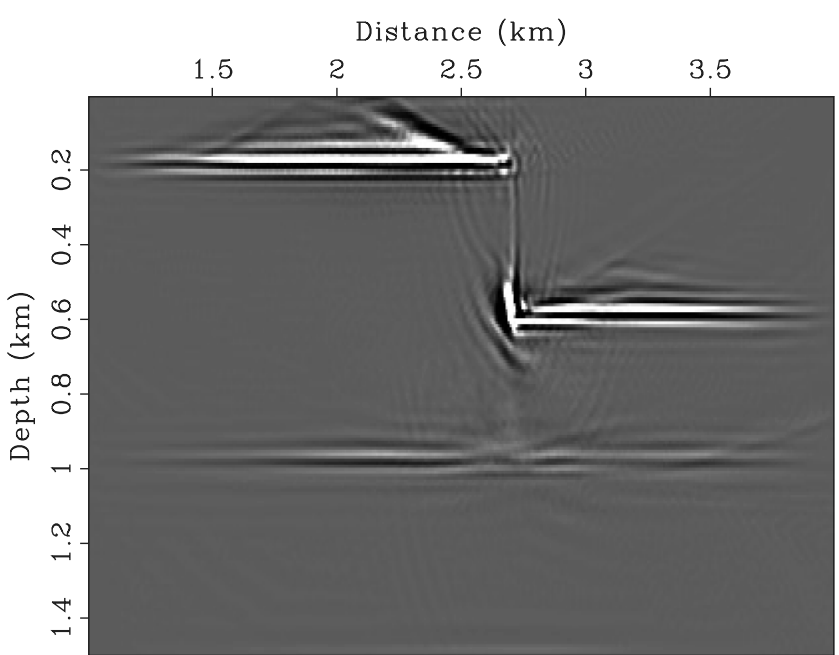

Figure 8. Sum of images in Figures 6 and 7.

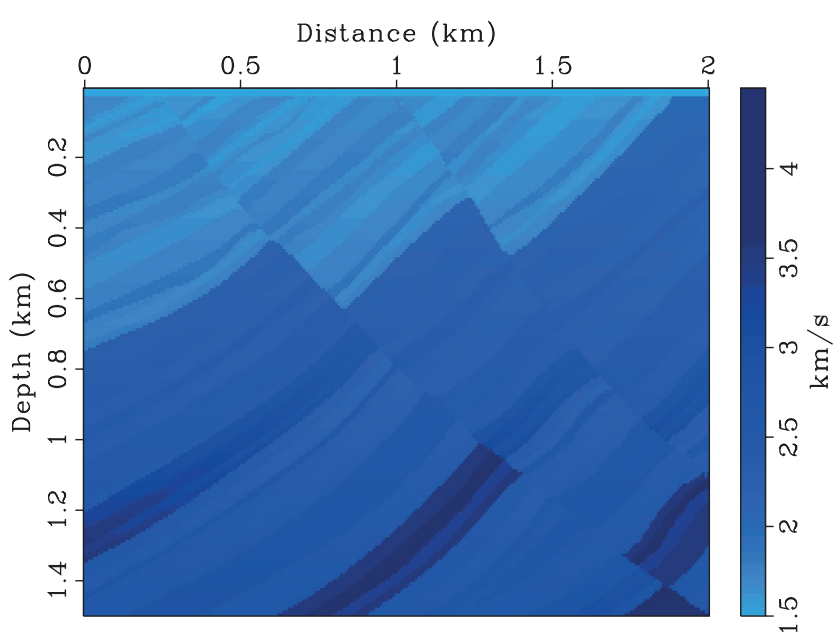

Figure 9. Part of the Marmousi model.

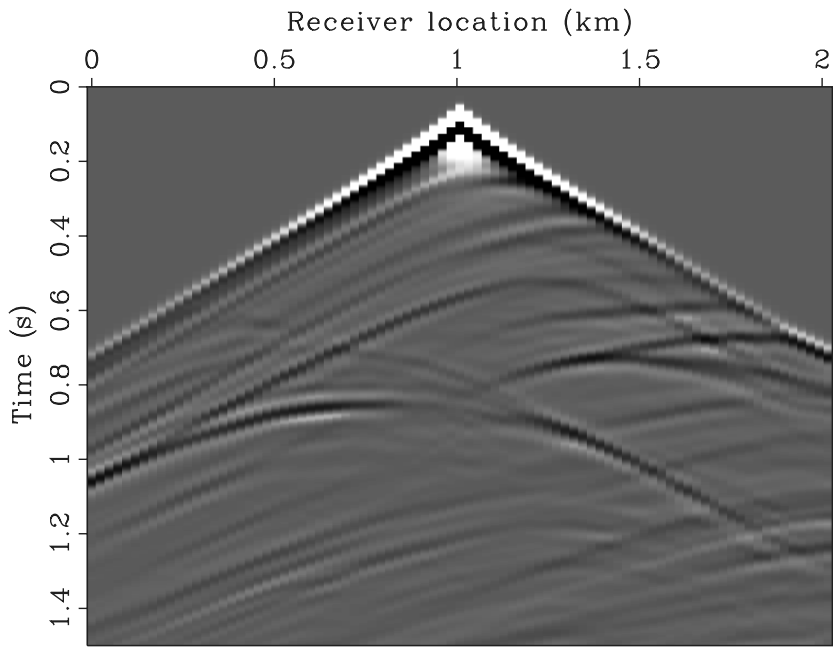

Figure 10. Source on the surface at horizontal location $1.0 \mathrm{~km}$.

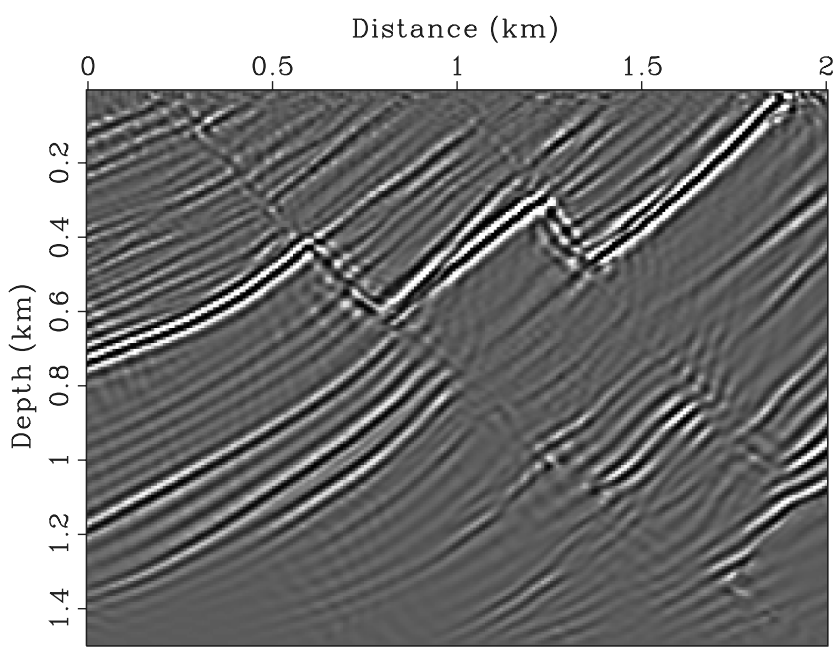

Figure 11. Single scattering image of the model in Figure 9.

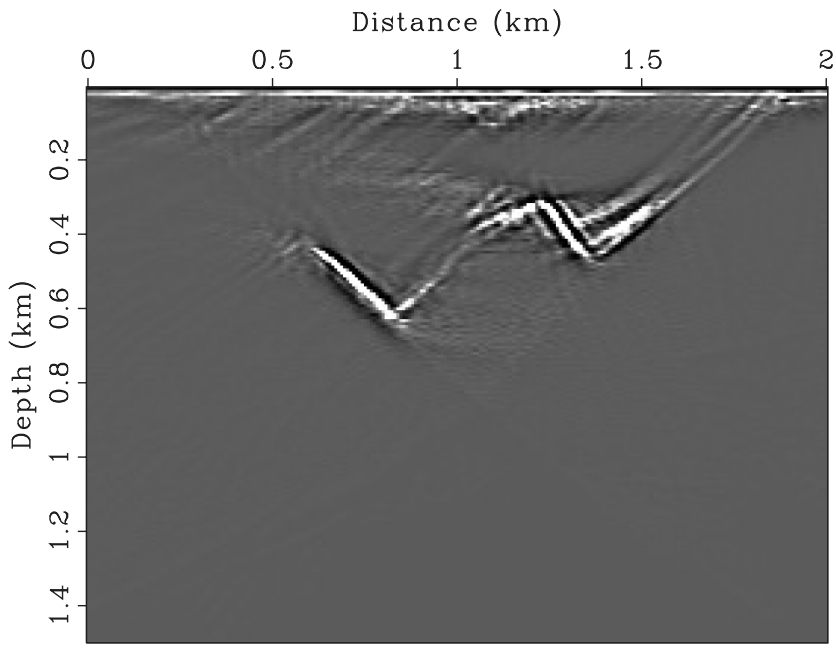

Figure 12. Image of the model in Figure 9 using the second-order Born scattering term.

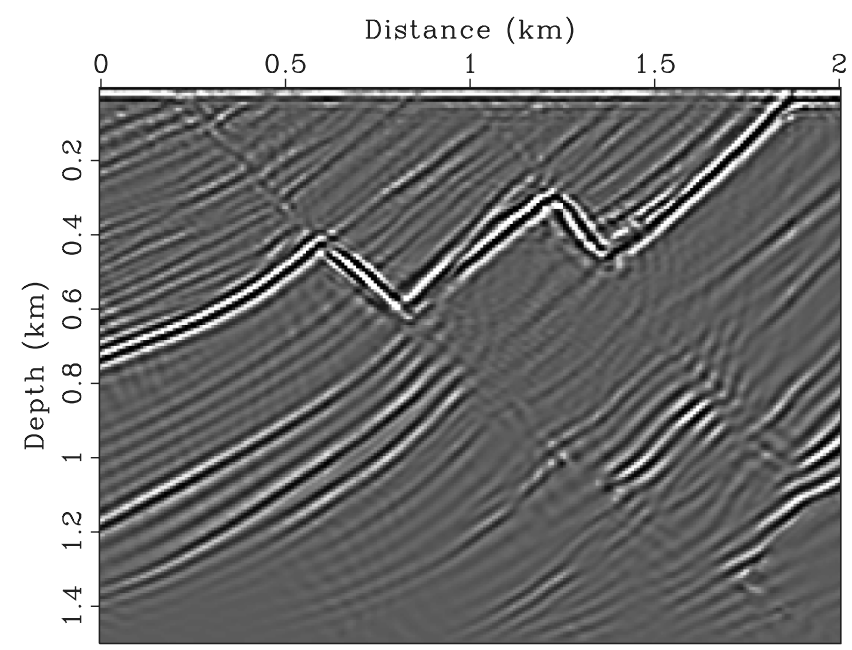

Figure 13. Sum of images in Figures 11 and 12. 


\section{DISCUSSION}

In the development of GIMI, we focused on introducing the process of enhancing various orders of internal multiples. We also showed that the first-order internal multiples are usually imaged using conventional RTM, but they do not contribute much considering their negligible amplitude when combined with single scattering. Moreover, the crosstalk resulting from their inaccurate imaging is still present and would become dominant if they occur in places in which there are no actual image points. With the process introduced here, we are able to isolate any order of internal multiples and image it. Like in the single-scattering case, we also end up with crosstalk that we tend to mitigate using a simple squaring of the image step, with an assumption that the noise will be lower in amplitude than the image. We are helped here by our objective of just mapping (imaging) the multiples, and, thus, the amplitudes are not treated accurately. An alternative, that we reserve for future work, is to implement this procedure within a least-squares migration approach. We expect that such an implementation will result in better amplitude treatment and better attenuation of the crosstalk noise.

The crosscorrelations described in GIMI resemble the crosscorrelations in Newton-Marchenko-Rose imaging (NMRI) proposed by Behura et al. (2012). Rather heuristically, one can say that in NMRI, internal multiples of all orders are generated and then imaged, whereas in GIMI, each order is generated independently and directly imaged. Theoretically, GIMI does not rely on the Marchenko equation; rather, the equations for GIMI are developed using seismic interferometry. Because we are using crosscorrelations instead of deconvolution, the amplitudes, like in conventional RTM, are not accurate and, therefore, strictly speaking, GIMI is not inverse scattering because the image we obtain does not give absolute strength of the scatterers.

Despite that the implementation here was done using integral methods of extrapolation (Kirchhoff), the approach is applicable to any type of extrapolation of the wavefield forward or backward. It is also not limited to acoustic or the type of wave modes available in our data because interferometry does not have these limitations.

\section{CONCLUSION}

We demonstrated that individual orders of internal multiples can be enhanced in imaging. This three-step procedure consists of first back propagating the recorded data using the background Green's function, followed by crosscorrelating the back-propagated wavefield with the surface data, and finally crosscorrelating the result with the forward-propagated source wavefield. As shown in the theory, this additional crosscorrelation between the surface recorded data and its back-propagated version enhances the second term of the Born scattering series (corresponding to the first-order internal multiple). To enhance the next-order multiple, we will need an additional crosscorrelation with the surface recorded data in step two of this three-step procedure. Therefore, in general, to enhance $N$ thorder term of the scattering series (or the $N-1$ order of internal multiple), we have to perform $N-1$ crosscorrelation operations. The crosscorrelations can be performed in the frequency domain, and the summations are only on the surface; therefore, the computational cost of each additional crosscorrelation is comparable with the Kirchhoff migration. In summary, we have proposed a method that is conceptually simple (perhaps algebraically tedious) and can easily be applied with a small addition to the computational cost. In this study, we have used a Kirchhoff-type implementation, which means that the computational cost for any order GIMI would be that of Kirchhoff migration plus the cost of the extra $N-1$ crosscorrelations.

\section{ACKNOWLEDGMENTS}

We thank KAUST for its financial support. We also thank C. Saragiotis for his help with coding.

\section{APPENDIX A}

\section{LEADING-ORDER TERMS FOR SECOND-ORDER INTERNAL MULTIPLES}

As discussed in the text, to image the third-order term in the Born scattering series (second-order internal multiples), there have to be $2(=3-1)$ crosscorrelations after the back propagation. This means that we have to crosscorrelate $G_{\text {back }}$ with $G_{s}$ two times. Therefore, we write

$$
\begin{aligned}
& \int_{\partial V} G_{s}\left(\mathbf{x}, \mathbf{x}_{1}\right)\left[\int_{\partial V} G_{s}\left(\mathbf{x}_{1}, \mathbf{x}_{0}\right) G_{\text {back }}^{*}\left(\mathbf{X}, \mathbf{x}_{0}\right) d^{2} \mathbf{x}_{0}\right]^{*} d^{2} \mathbf{x}_{1} \approx \\
& \omega^{4} \int_{\partial V} G_{s}\left(\mathbf{x}, \mathbf{x}_{1}\right)\left[\int_{V} \int_{V} \delta v\left(\mathbf{x}^{\prime}\right) \delta v\left(\mathbf{x}^{\prime \prime}\right) G_{0}\left(\mathbf{x}_{1}, \mathbf{x}^{\prime \prime}\right) G_{0}\left(\mathbf{x}^{\prime \prime}, \mathbf{x}^{\prime}\right) G_{0}\left(\mathbf{x}^{\prime}, \mathbf{X}\right) d^{3} \mathbf{x}^{\prime} d^{3} \mathbf{x}^{\prime \prime}\right]^{*} d^{2} \mathbf{x}_{1} \\
& +\omega^{4} \int_{\partial V} G_{s}\left(\mathbf{x}, \mathbf{x}_{1}\right)\left[\int_{V} \int_{V} \delta v\left(\mathbf{x}^{\prime}\right) \delta v\left(\mathbf{x}^{\prime \prime}\right) G_{0}\left(\mathbf{x}_{1}, \mathbf{x}^{\prime \prime}\right) G_{0}^{*}\left(\mathbf{x}^{\prime \prime}, \mathbf{x}^{\prime}\right) G_{0}\left(\mathbf{x}^{\prime}, \mathbf{X}\right) d^{3} \mathbf{x}^{\prime} d^{3} \mathbf{x}^{\prime \prime}\right]^{*} d^{2} \mathbf{x}_{1} \\
& +\omega^{4} \int_{\partial V} G_{s}\left(\mathbf{x}, \mathbf{x}_{1}\right)\left[\int_{V} \int_{V} \delta v\left(\mathbf{x}^{\prime}\right) \delta v\left(\mathbf{x}^{\prime \prime}\right) G_{0}\left(\mathbf{x}_{1}, \mathbf{x}^{\prime \prime}\right) G_{0}\left(\mathbf{x}^{\prime \prime}, \mathbf{x}^{\prime}\right) G_{0}^{*}\left(\mathbf{x}^{\prime}, \mathbf{X}\right) d^{3} \mathbf{x}^{\prime} d^{3} \mathbf{x}^{\prime \prime}\right]^{*} d^{2} \mathbf{x}_{1} \\
& +\omega^{4} \int_{\partial V} G_{s}\left(\mathbf{x}, \mathbf{x}_{1}\right)\left[\int_{V} \int_{V} \delta v\left(\mathbf{x}^{\prime}\right) \delta v\left(\mathbf{x}^{\prime \prime}\right) G_{0}\left(\mathbf{x}_{1}, \mathbf{x}^{\prime \prime}\right) G_{0}^{*}\left(\mathbf{x}^{\prime \prime}, \mathbf{x}^{\prime}\right) G_{0}^{*}\left(\mathbf{x}^{\prime}, \mathbf{X}\right) d^{3} \mathbf{x}^{\prime} d^{3} \mathbf{x}^{\prime \prime}\right]^{*} d^{2} \mathbf{x}_{1} \\
& +\ldots
\end{aligned}
$$

Using equations 1 and 2 in equation $\mathrm{A}-1$, we get

$$
\begin{aligned}
& \int_{\partial V} G_{s}\left(\mathbf{x}, \mathbf{x}_{1}\right)\left[\int_{\partial V} G_{s}\left(\mathbf{x}_{1}, \mathbf{x}_{0}\right) G_{\text {back }}^{*}\left(\mathbf{X}, \mathbf{x}_{0}\right) d^{2} \mathbf{x}_{0}\right]^{*} d^{2} \mathbf{x}_{1} \approx \\
& \omega^{6} \int_{V} \int_{V} \int_{V} \delta v\left(\mathbf{x}^{\prime}\right) \delta v\left(\mathbf{x}^{\prime \prime}\right) \delta v\left(\mathbf{x}^{\prime \prime \prime}\right) G_{0}\left(\mathbf{x}, \mathbf{x}^{\prime \prime \prime}\right) G_{0}\left(\mathbf{x}^{\prime \prime \prime}, \mathbf{x}^{\prime \prime}\right) G_{0}^{*}\left(\mathbf{x}^{\prime \prime}, \mathbf{x}^{\prime}\right) G_{0}^{*}\left(\mathbf{x}^{\prime}, \mathbf{X}\right) d^{3} \mathbf{x}^{\prime} d^{3} \mathbf{x}^{\prime \prime} d^{3} \mathbf{x}^{\prime \prime \prime} \\
& +\omega^{6} \int_{V} \int_{V} \int_{V} \delta v\left(\mathbf{x}^{\prime}\right) \delta v\left(\mathbf{x}^{\prime \prime}\right) \delta v\left(\mathbf{x}^{\prime \prime \prime}\right) G_{0}\left(\mathbf{x}, \mathbf{x}^{\prime \prime \prime}\right) G_{0}\left(\mathbf{x}^{\prime \prime \prime}, \mathbf{x}^{\prime \prime}\right) G_{0}\left(\mathbf{x}^{\prime \prime}, \mathbf{x}^{\prime}\right) G_{0}^{*}\left(\mathbf{x}^{\prime}, \mathbf{X}\right) d^{3} \mathbf{x}^{\prime} d^{3} \mathbf{x}^{\prime \prime} d^{3} \mathbf{x}^{\prime \prime \prime} \\
& +\omega^{6} \int_{V} \int_{V} \int_{V} \delta v\left(\mathbf{x}^{\prime}\right) \delta v\left(\mathbf{x}^{\prime \prime}\right) \delta v\left(\mathbf{x}^{\prime \prime \prime}\right) G_{0}\left(\mathbf{x}, \mathbf{x}^{\prime \prime \prime}\right) G_{0}\left(\mathbf{x}^{\prime \prime \prime}, \mathbf{x}^{\prime \prime}\right) G_{0}^{*}\left(\mathbf{x}^{\prime \prime}, \mathbf{x}^{\prime}\right) G_{0}\left(\mathbf{x}^{\prime}, \mathbf{X}\right) d^{3} \mathbf{x}^{\prime} d^{3} \mathbf{x}^{\prime \prime} d^{3} \mathbf{x}^{\prime \prime \prime} \\
& +\omega^{6} \int_{V} \int_{V} \int_{V} \delta v\left(\mathbf{x}^{\prime}\right) \delta v\left(\mathbf{x}^{\prime \prime}\right) \delta v\left(\mathbf{x}^{\prime \prime \prime}\right) G_{0}\left(\mathbf{x}, \mathbf{x}^{\prime \prime \prime}\right) G_{0}\left(\mathbf{x}^{\prime \prime \prime}, \mathbf{x}^{\prime \prime}\right) G_{0}\left(\mathbf{x}^{\prime \prime}, \mathbf{x}^{\prime}\right) G_{0}\left(\mathbf{x}^{\prime}, \mathbf{X}\right) d^{3} \mathbf{x}^{\prime} d^{3} \mathbf{x}^{\prime \prime} d^{3} \mathbf{x}^{\prime \prime \prime} \\
& +\omega^{6} \int_{V} \int_{V} \int_{V} \delta v\left(\mathbf{x}^{\prime}\right) \delta v\left(\mathbf{x}^{\prime \prime}\right) \delta v\left(\mathbf{x}^{\prime \prime \prime}\right) G_{0}\left(\mathbf{x}, \mathbf{x}^{\prime \prime \prime}\right) G_{0}^{*}\left(\mathbf{x}^{\prime \prime \prime}, \mathbf{x}^{\prime \prime}\right) G_{0}^{*}\left(\mathbf{x}^{\prime \prime}, \mathbf{x}^{\prime}\right) G_{0}^{*}\left(\mathbf{x}^{\prime}, \mathbf{X}\right) d^{3} \mathbf{x}^{\prime} d^{3} \mathbf{x}^{\prime \prime} d^{3} \mathbf{x}^{\prime \prime \prime} \\
& \left.\left.+\omega_{V} \int_{V} \delta v\left(\mathbf{x}^{\prime}\right) \delta v\left(\mathbf{x}_{V} \int_{V} \int_{V} \delta v\left(\mathbf{x}^{\prime}\right) \delta v\left(\mathbf{x}^{\prime \prime}\right) \delta v\left(\mathbf{x}^{\prime \prime \prime \prime}\right) G_{0}\left(\mathbf{x}, \mathbf{x}^{\prime \prime \prime}\right) G_{0}\left(\mathbf{x}, \mathbf{x}^{\prime \prime \prime}\right) G_{0}^{*}\left(\mathbf{x}^{\prime \prime \prime}, \mathbf{x}^{\prime \prime \prime}\right) \mathbf{x}_{0}\left(\mathbf{x}^{\prime \prime}\right) G_{0}^{*}, \mathbf{x}^{\prime}\right) G_{0}^{*}, \mathbf{x}^{\prime}, \mathbf{X}\right) d^{3}\right) \mathbf{x}_{0}\left(\mathbf{x}^{\prime}, d^{3} \mathbf{x}^{\prime \prime} d^{3} \mathbf{x}^{\prime \prime \prime} \mathbf{x}^{\prime} \mathbf{x}^{\prime} d^{3} \mathbf{x}^{\prime \prime} d^{3} \mathbf{x}^{\prime \prime \prime}\right. \\
& +\omega^{6} \int_{V} \int_{V} \int_{V} \delta v\left(\mathbf{x}^{\prime}\right) \delta v\left(\mathbf{x}^{\prime \prime}\right) \delta v\left(\mathbf{x}^{\prime \prime \prime}\right) G_{0}\left(\mathbf{x}, \mathbf{x}^{\prime \prime \prime}\right) G_{0}^{*}\left(\mathbf{x}^{\prime \prime \prime}, \mathbf{x}^{\prime \prime}\right) G_{0}\left(\mathbf{x}^{\prime \prime}, \mathbf{x}^{\prime}\right) G_{0}\left(\mathbf{x}^{\prime}, \mathbf{X}\right) d^{3} \mathbf{x}^{\prime} d^{3} \mathbf{x}^{\prime \prime} d^{3} \mathbf{x}^{\prime \prime \prime} \\
& +\ldots
\end{aligned}
$$

Then, for the imaging step, we crosscorrelate equation A-2 by the background Green's function from the surface to the image point. Again, we use equation 2 and get 16 terms for the leading order, i.e., 


$$
\begin{aligned}
& \mathbf{I}_{3} \equiv \int_{\partial V} G_{0}(\mathbf{X}, \mathbf{x})\left[\int_{\partial V} G_{s}\left(\mathbf{x}, \mathbf{x}_{1}\right)\left[\int_{\partial V} G_{s}\left(\mathbf{x}_{1}, \mathbf{x}_{0}\right) G_{\text {back }}^{*}\left(\mathbf{X}, \mathbf{x}_{0}\right) d^{2} \mathbf{x}_{0}\right]^{*} d^{2} \mathbf{x}_{1}\right]^{*} d^{2} \mathbf{x} \approx \\
& \omega^{6} \int_{V} \int_{V} \int_{V} \delta v\left(\mathbf{x}^{\prime}\right) \delta v\left(\mathbf{x}^{\prime \prime}\right) \delta v\left(\mathbf{x}^{\prime \prime \prime}\right) G_{0}\left(\mathbf{X}, \mathbf{x}^{\prime \prime \prime}\right) G_{0}^{*}\left(\mathbf{x}^{\prime \prime \prime}, \mathbf{x}^{\prime \prime}\right) G_{0}\left(\mathbf{x}^{\prime \prime}, \mathbf{x}^{\prime}\right) G_{0}\left(\mathbf{x}^{\prime}, \mathbf{X}\right) d^{3} \mathbf{x}^{\prime} d^{3} \mathbf{x}^{\prime \prime} d^{3} \mathbf{x}^{\prime \prime \prime} \\
& +\omega^{6} \int_{V} \int_{V} \int_{V} \delta v\left(\mathbf{x}^{\prime}\right) \delta v\left(\mathbf{x}^{\prime \prime}\right) \delta v\left(\mathbf{x}^{\prime \prime \prime}\right) G_{0}\left(\mathbf{X}, \mathbf{x}^{\prime \prime \prime}\right) G_{0}^{*}\left(\mathbf{x}^{\prime \prime \prime}, \mathbf{x}^{\prime \prime}\right) G_{0}^{*}\left(\mathbf{x}^{\prime \prime}, \mathbf{x}^{\prime}\right) G_{0}\left(\mathbf{x}^{\prime}, \mathbf{X}\right) d^{3} \mathbf{x}^{\prime} d^{3} \mathbf{x}^{\prime \prime} d^{3} \mathbf{x}^{\prime \prime \prime} \\
& +\omega^{6} \int_{V} \int_{V} \int_{V} \delta v\left(\mathbf{x}^{\prime}\right) \delta v\left(\mathbf{x}^{\prime \prime}\right) \delta v\left(\mathbf{x}^{\prime \prime \prime}\right) G_{0}\left(\mathbf{X}, \mathbf{x}^{\prime \prime \prime}\right) G_{0}^{*}\left(\mathbf{x}^{\prime \prime \prime}, \mathbf{x}^{\prime \prime}\right) G_{0}\left(\mathbf{x}^{\prime \prime}, \mathbf{x}^{\prime}\right) G_{0}^{*}\left(\mathbf{x}^{\prime}, \mathbf{X}\right) d^{3} \mathbf{x}^{\prime} d^{3} \mathbf{x}^{\prime \prime} d^{3} \mathbf{x}^{\prime \prime \prime} \\
& +\omega^{6} \int_{V} \int_{V} \int_{V} \delta v\left(\mathbf{x}^{\prime}\right) \delta v\left(\mathbf{x}^{\prime \prime}\right) \delta v\left(\mathbf{x}^{\prime \prime \prime}\right) G_{0}\left(\mathbf{X}, \mathbf{x}^{\prime \prime \prime}\right) G_{0}^{*}\left(\mathbf{x}^{\prime \prime \prime}, \mathbf{x}^{\prime \prime}\right) G_{0}^{*}\left(\mathbf{x}^{\prime \prime}, \mathbf{x}^{\prime}\right) G_{0}^{*}\left(\mathbf{x}^{\prime}, \mathbf{X}\right) d^{3} \mathbf{x}^{\prime} d^{3} \mathbf{x}^{\prime \prime} d^{3} \mathbf{x}^{\prime \prime \prime} \\
& +\omega^{6} \int_{V} \int_{V} \int_{V} \delta v\left(\mathbf{x}^{\prime}\right) \delta v\left(\mathbf{x}^{\prime \prime}\right) \delta v\left(\mathbf{x}^{\prime \prime \prime}\right) G_{0}\left(\mathbf{X}, \mathbf{x}^{\prime \prime \prime}\right) G_{0}\left(\mathbf{x}^{\prime \prime \prime}, \mathbf{x}^{\prime \prime}\right) G_{0}\left(\mathbf{x}^{\prime \prime}, \mathbf{x}^{\prime}\right) G_{0}\left(\mathbf{x}^{\prime}, \mathbf{X}\right) d^{3} \mathbf{x}^{\prime} d^{3} \mathbf{x}^{\prime \prime} d^{3} \mathbf{x}^{\prime \prime \prime} \\
& +\omega^{6} \int_{V} \int_{V} \int_{V} \delta v\left(\mathbf{x}^{\prime}\right) \delta v\left(\mathbf{x}^{\prime \prime}\right) \delta v\left(\mathbf{x}^{\prime \prime \prime}\right) G_{0}\left(\mathbf{X}, \mathbf{x}^{\prime \prime \prime}\right) G_{0}\left(\mathbf{x}^{\prime \prime \prime}, \mathbf{x}^{\prime \prime}\right) G_{0}^{*}\left(\mathbf{x}^{\prime \prime}, \mathbf{x}^{\prime}\right) G_{0}\left(\mathbf{x}^{\prime}, \mathbf{X}\right) d^{3} \mathbf{x}^{\prime} d^{3} \mathbf{x}^{\prime \prime} d^{3} \mathbf{x}^{\prime \prime \prime} \\
& +\omega^{6} \int_{V} \int_{V} \int_{V} \delta v\left(\mathbf{x}^{\prime}\right) \delta v\left(\mathbf{x}^{\prime \prime}\right) \delta v\left(\mathbf{x}^{\prime \prime \prime}\right) G_{0}\left(\mathbf{X}, \mathbf{x}^{\prime \prime \prime}\right) G_{0}\left(\mathbf{x}^{\prime \prime \prime}, \mathbf{x}^{\prime \prime}\right) G_{0}\left(\mathbf{x}^{\prime \prime}, \mathbf{x}^{\prime}\right) G_{0}^{*}\left(\mathbf{x}^{\prime}, \mathbf{X}\right) d^{3} \mathbf{x}^{\prime} d^{3} \mathbf{x}^{\prime \prime} d^{3} \mathbf{x}^{\prime \prime \prime} \\
& +\omega^{6} \int_{V} \int_{V} \int_{V} \delta v\left(\mathbf{x}^{\prime}\right) \delta v\left(\mathbf{x}^{\prime \prime}\right) \delta v\left(\mathbf{x}^{\prime \prime \prime}\right) G_{0}\left(\mathbf{X}, \mathbf{x}^{\prime \prime \prime}\right) G_{0}\left(\mathbf{x}^{\prime \prime \prime}, \mathbf{x}^{\prime \prime}\right) G_{0}^{*}\left(\mathbf{x}^{\prime \prime}, \mathbf{x}^{\prime}\right) G_{0}^{*}\left(\mathbf{x}^{\prime}, \mathbf{X}\right) d^{3} \mathbf{x}^{\prime} d^{3} \mathbf{x}^{\prime \prime} d^{3} \mathbf{x}^{\prime \prime \prime} \\
& +\omega^{6} \int_{V} \int_{V} \int_{V} \delta v\left(\mathbf{x}^{\prime}\right) \delta v\left(\mathbf{x}^{\prime \prime}\right) \delta v\left(\mathbf{x}^{\prime \prime \prime}\right) G_{0}^{*}\left(\mathbf{X}, \mathbf{x}^{\prime \prime \prime}\right) G_{0}^{*}\left(\mathbf{x}^{\prime \prime \prime}, \mathbf{x}^{\prime \prime}\right) G_{0}\left(\mathbf{x}^{\prime \prime}, \mathbf{x}^{\prime}\right) G_{0}\left(\mathbf{x}^{\prime}, \mathbf{X}\right) d^{3} \mathbf{x}^{\prime} d^{3} \mathbf{x}^{\prime \prime} d^{3} \mathbf{x}^{\prime \prime \prime} \\
& +\omega^{6} \int_{V} \int_{V} \int_{V} \delta v\left(\mathbf{x}^{\prime}\right) \delta v\left(\mathbf{x}^{\prime \prime}\right) \delta v\left(\mathbf{x}^{\prime \prime \prime}\right) G_{0}^{*}\left(\mathbf{X}, \mathbf{x}^{\prime \prime \prime}\right) G_{0}^{*}\left(\mathbf{x}^{\prime \prime \prime}, \mathbf{x}^{\prime \prime}\right) G_{0}^{*}\left(\mathbf{x}^{\prime \prime}, \mathbf{x}^{\prime}\right) G_{0}\left(\mathbf{x}^{\prime}, \mathbf{X}\right) d^{3} \mathbf{x}^{\prime} d^{3} \mathbf{x}^{\prime \prime} d^{3} \mathbf{x}^{\prime \prime \prime} \\
& +\omega^{6} \int_{V} \int_{V} \int_{V} \delta v\left(\mathbf{x}^{\prime}\right) \delta v\left(\mathbf{x}^{\prime \prime}\right) \delta v\left(\mathbf{x}^{\prime \prime \prime}\right) G_{0}^{*}\left(\mathbf{X}, \mathbf{x}^{\prime \prime \prime}\right) G_{0}^{*}\left(\mathbf{x}^{\prime \prime \prime}, \mathbf{x}^{\prime \prime}\right) G_{0}\left(\mathbf{x}^{\prime \prime}, \mathbf{x}^{\prime}\right) G_{0}^{*}\left(\mathbf{x}^{\prime}, \mathbf{X}\right) d^{3} \mathbf{x}^{\prime} d^{3} \mathbf{x}^{\prime \prime} d^{3} \mathbf{x}^{\prime \prime \prime} \\
& +\omega^{6} \int_{V} \int_{V} \int_{V} \delta v\left(\mathbf{x}^{\prime}\right) \delta v\left(\mathbf{x}^{\prime \prime}\right) \delta v\left(\mathbf{x}^{\prime \prime \prime}\right) G_{0}^{*}\left(\mathbf{X}, \mathbf{x}^{\prime \prime \prime}\right) G_{0}^{*}\left(\mathbf{x}^{\prime \prime \prime}, \mathbf{x}^{\prime \prime}\right) G_{0}^{*}\left(\mathbf{x}^{\prime \prime}, \mathbf{x}^{\prime}\right) G_{0}^{*}\left(\mathbf{x}^{\prime}, \mathbf{X}\right) d^{3} \mathbf{x}^{\prime} d^{3} \mathbf{x}^{\prime \prime} d^{3} \mathbf{x}^{\prime \prime \prime} \\
& +\omega^{6} \int_{V} \int_{V} \int_{V} \delta v\left(\mathbf{x}^{\prime}\right) \delta v\left(\mathbf{x}^{\prime \prime}\right) \delta v\left(\mathbf{x}^{\prime \prime \prime}\right) G_{0}^{*}\left(\mathbf{X}, \mathbf{x}^{\prime \prime \prime}\right) G_{0}\left(\mathbf{x}^{\prime \prime \prime}, \mathbf{x}^{\prime \prime}\right) G_{0}\left(\mathbf{x}^{\prime \prime}, \mathbf{x}^{\prime}\right) G_{0}\left(\mathbf{x}^{\prime}, \mathbf{X}\right) d^{3} \mathbf{x}^{\prime} d^{3} \mathbf{x}^{\prime \prime} d^{3} \mathbf{x}^{\prime \prime \prime} \\
& +\omega^{6} \int_{V} \int_{V} \int_{V} \delta v\left(\mathbf{x}^{\prime}\right) \delta v\left(\mathbf{x}^{\prime \prime}\right) \delta v\left(\mathbf{x}^{\prime \prime \prime}\right) G_{0}^{*}\left(\mathbf{X}, \mathbf{x}^{\prime \prime \prime}\right) G_{0}\left(\mathbf{x}^{\prime \prime \prime}, \mathbf{x}^{\prime \prime}\right) G_{0}^{*}\left(\mathbf{x}^{\prime \prime}, \mathbf{x}^{\prime}\right) G_{0}\left(\mathbf{x}^{\prime}, \mathbf{X}\right) d^{3} \mathbf{x}^{\prime} d^{3} \mathbf{x}^{\prime \prime} d^{3} \mathbf{x}^{\prime \prime \prime} \\
& +\omega^{6} \int_{V} \int_{V} \int_{V} \delta v\left(\mathbf{x}^{\prime}\right) \delta v\left(\mathbf{x}^{\prime \prime}\right) \delta v\left(\mathbf{x}^{\prime \prime \prime}\right) G_{0}^{*}\left(\mathbf{X}, \mathbf{x}^{\prime \prime \prime}\right) G_{0}\left(\mathbf{x}^{\prime \prime \prime}, \mathbf{x}^{\prime \prime}\right) G_{0}\left(\mathbf{x}^{\prime \prime}, \mathbf{x}^{\prime}\right) G_{0}^{*}\left(\mathbf{x}^{\prime}, \mathbf{X}\right) d^{3} \mathbf{x}^{\prime} d^{3} \mathbf{x}^{\prime \prime} d^{3} \mathbf{x}^{\prime \prime \prime} \\
& +\omega^{6} \int_{V} \int_{V} \int_{V} \delta v\left(\mathbf{x}^{\prime}\right) \delta v\left(\mathbf{x}^{\prime \prime}\right) \delta v\left(\mathbf{x}^{\prime \prime \prime}\right) G_{0}^{*}\left(\mathbf{X}, \mathbf{x}^{\prime \prime \prime}\right) G_{0}\left(\mathbf{x}^{\prime \prime \prime}, \mathbf{x}^{\prime \prime}\right) G_{0}^{*}\left(\mathbf{x}^{\prime \prime}, \mathbf{x}^{\prime}\right) G_{0}^{*}\left(\mathbf{x}^{\prime}, \mathbf{X}\right) d^{3} \mathbf{x}^{\prime} d^{3} \mathbf{x}^{\prime \prime} d^{3} \mathbf{x}^{\prime \prime \prime} \\
& +\ldots \text {. }
\end{aligned}
$$

The 16 terms on the right side of equation A-3 can be analyzed in a similar fashion as the other lower order multiple images. The first eight terms are the complex conjugate of the last eight, and different terms will image different points (as for the terms in equation 11).

\section{REFERENCES}

Behura, J., K. Wapenaar, and R. Snieder, 2012, Newton-Marchenko-Rose imaging: SEG.

Behura, J., K. Wapenaar, and R. Snieder, 2014, Autofocus imaging: Image reconstruction based on inverse scattering theory: Geophysics, 79, no. 3, A19-A26, doi: 10.1190/geo2013-0398.1.

Ikelle, L. T., and L. Amundsen, 2005, Introduction to petroleum seismology: SEG.

Malcolm, A., M. De Hoop, and B. Ursin, 2011, Recursive imaging with multiply scattered waves using partial image regularization: A North Sea case study: Geophysics, 76, no. 2, B33-B42, doi: 10.1190/1.3537822.

Malcolm, A. E., B. Ursin, and M. V. De Hoop, 2009, Seismic imaging and illumination with internal multiples: Geophysical Journal International, 176, 847-864, doi: 10.1111/j.1365-246X.2008.03992.x.

Schuster, G. T., J. Yu, J. Sheng, and J. Rickett, 2004, Interferometric/daylight seismic imaging: Geophysical Journal International, 157, 838-852, doi: 10.1111/j.1365-246X.2004.02251.x.

Shan, G., and A. Guitton, 2004, Migration of surface related multiples: Tests on the Sigsbee2B data set: SEG.

Slob, E., K. Wapenaar, F. Broggini, and R. Snieder, 2014, Seismic reflector imaging using internal multiples with Marchenko-type equations: Geophysics, 79, no. 2, S63-S76, doi: 10.1190/geo2013-0095.1.

Wapenaar, K., and J. Fokkema, 2006, Green's function representations for seismic interferometry: Geophysics, 71, no. 4, SI33-SI46, doi: 10.1190/1 .2213955

Wapenaar, K., E. Slob, and R. Snieder, 2010, On seismic interferometry, the generalized optical theorem, and the scattering matrix of a point scatterer: Geophysics, 75, no. 3, SA27-SA35, doi: 10.1190/1.3374359.

Wong, M., B. Biondi, and S. Ronen, 2012, Imaging with multiples using linearized full-wave inversion: SEG

Wong, M., S. Ronen, and B. Biondi, 2011, Least-squares reverse time migration/inversion for ocean bottom data: A case study: SEG.

Zhang, Y., and J. Sun, 2009, Practical issues in reverse time migration: True amplitude gathers, noise removal and harmonic source encoding: First Break, 27, 53-60, doi: 10.3997/1365-2397.2009002.

Zuberi, A., and T. Alkhalifah, 2013, Imaging by forward propagating the data: Theory and application: Geophysical Prospecting, 61, 248-267, doi: 10.1111/1365-2478.12006.

Zuberi, M. A. H., and T. Alkhalifah, 2014, Generalized internal multiple imaging (GIMI) using Feynman-like diagrams: Geophysical Journal International, 197, 1582-1592, doi: 10.1093/gji/ggt527. 